\title{
11. LOW-TEMPERATURE ALTERATION OF BASALTS FROM THE HAWAIIAN ARCH, LEG 136 $^{1}$
}

\author{
Jeffrey C. Alt ${ }^{2}$
}

\begin{abstract}
The mineralogy and chemistry of altered basalts and the stable isotopic compositions of secondary vein carbonates were studied in cores from Ocean Drilling Program Hole 843B, located in 95-Ma crust of the Hawaiian Arch. Millimeter- to centimeter-sized dark alteration halos around veins are $5 \%-15 \%$ altered to celadonite and Fe-oxyhydroxides, plus minor saponite and calcite. Adjacent gray host rocks are about $15 \%$ altered to saponite and calcite. The dark halos are enriched in $\mathrm{H}_{2} \mathrm{O}^{+}, \mathrm{CO}_{2}$, $\mathrm{Fe}^{\mathrm{T}}, \mathrm{K}_{2} \mathrm{O}, \mathrm{MnO}$, and $\mathrm{Fe}^{3+} / \mathrm{Fe}^{\mathrm{T}}$ and depleted in $\mathrm{SiO}_{2}, \mathrm{Al}_{2} \mathrm{O}_{3}, \mathrm{MgO}$, and $\mathrm{TiO}_{2}$ relative to gray host rocks. Brown alteration halos occur around veins where veins are more abundant, and are similar to dark halos, but contain more Fe-oxyhydroxides and exhibit greater $\mathrm{Fe}_{2} \mathrm{O}_{3}{ }^{\mathrm{T}}$ contents and higher $\mathrm{Fe}^{3+} / \mathrm{Fe}^{\mathrm{T}}$. Stable isotopic compositions of vein carbonates are consistent with their precipitation from seawater at temperatures of $5^{\circ}-40^{\circ} \mathrm{C}$. Crosscutting relationships of veins and zoned vein and vesicle fillings reveal a sequence of secondary mineral formation and alteration conditions. Celadonite and Fe-oxyhydroxides formed and dark alteration halos developed relatively early, under oxidizing conditions at low temperatures $\left(<50^{\circ} \mathrm{C}\right)$. Saponite formed later at lower seawater/rock ratios and under more reducing conditions. Calcite and pyrite formed last in veins and vesicles from more evolved, seawaterderived fluids at temperatures of $5^{\circ}-40^{\circ} \mathrm{C}$. A second stage of celadonite, with compositions distinct from the early celadonite, also occurred relatively late (within the "calcite stage"), and may be related to refracturing of the crust and introduction of less-evolved seawater solutions into the rocks. Trends to higher $\mathrm{K}_{2} \mathrm{O}$ contents are attributed to alteration, but high $\mathrm{K} / \mathrm{Ti}$, $\mathrm{Ba}$, and $\mathrm{Zr}$ contents indicate the presence of enriched or transitional MORB. $\mathrm{CO}_{2}$ contents of Pacific ODP cores exhibit a general increase with age suggesting progressive fixation of $\mathrm{CO}_{2}$ as calcite in the crust, but this could be complicated by local heterogeneities in fracturing and calcite formation in the crust.
\end{abstract}

\section{INTRODUCTION}

The main objective of Ocean Drilling Program (ODP) Leg 136 was to establish an Ocean Seismograph Network (OSN) site on the Hawaiian Arch southwest of Hawaii. This site is to be used for borehole seismometer experiments as part of a larger global network to study such topics as earth structure, upper mantle dynamics, earthquake sources, ocean crustal structure, and seismic noise. Because the physical properties of the crust can be significantly affected by alteration of the rocks and cementation of fractures, understanding how the crust is altered is an important part of these goals. The crust of the Hawaiian Arch, where the OSN site is located, is the material on which the Hawaiian Islands are built and through which Hawaiian lavas were first erupted. A secondary goal of Leg 136 was to determine the composition of this crust and constrain its role in contamination of Hawaiian magmas. Significant chemical and mineralogical changes can occur through interaction of basement with seawater, so understanding these changes is an important part of determining the overall composition of the crust.

This study presents the mineralogy and chemistry of altered basement rocks, plus stable isotopic data for vein carbonates in the rocks, from the OSN site drilled during Leg 136. The purpose of this work is to understand the processes responsible for alteration of the upper crust at the site, and what chemical changes have occurred in the rocks as the result. Results for this site are also compared with data for both younger and older Pacific ocean crust to determine if trends due to progressive aging of the crust can be recognized.

\section{Hole 843B}

Hole $843 \mathrm{~B}$ is located on the Hawaiian Arch, about $225 \mathrm{~km}$ southwest of the island of Oahu, at a water depth of $4407.1 \mathrm{~m}$ (Fig. 1). Preliminary paleontologic dating places the age of the crust at about

' Wilkens, R.H., Firth, J., Bender, J., et al., 1993. Proc. ODP, Sci. Results, 136: College Station, TX (Ocean Drilling Program).

${ }^{2}$ Department of Geological Sciences, 1006 C.C. Little Building. The University of Michigan, Ann Arbor, MI 48109-1063, U.S.A.

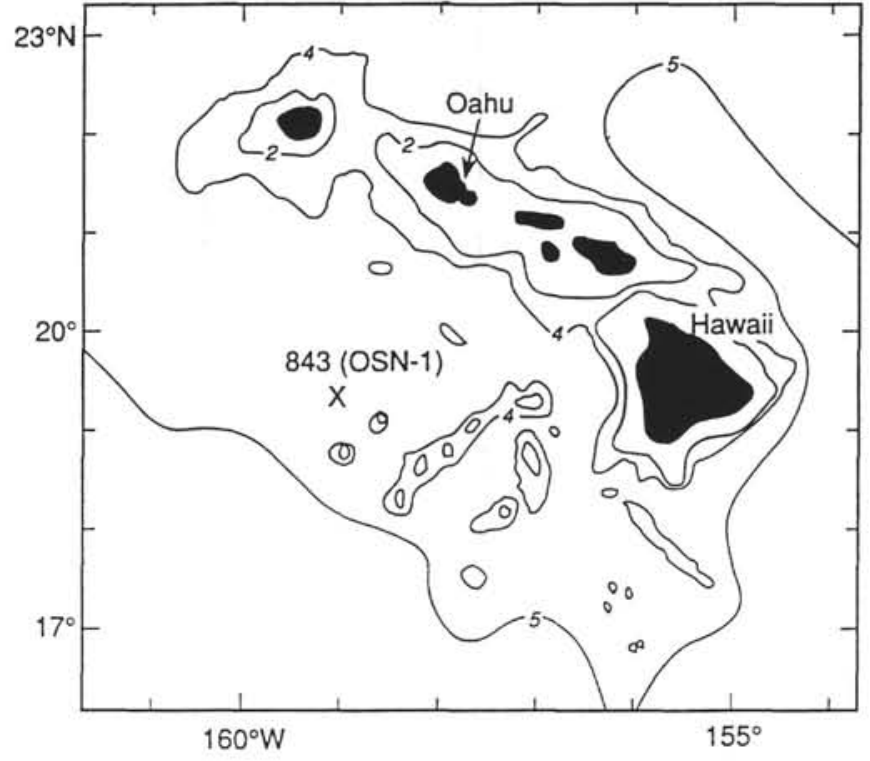

Figure 1. Location of Site 843 southwest of the island of Oahu. Water depth contours in kilometers.

$95 \mathrm{Ma}$ (Dziewonski, Wilkens, Firth, et al., 1992). Sedimentation rates from 100 to $80 \mathrm{Ma}$ averaged about $3 \mathrm{~m} / \mathrm{m}$.y. (Winterer, Ewing, et al., 1973). The hole penetrates through $242.5 \mathrm{~m}$ of sediment and $70.9 \mathrm{~m}$ into basement, to a total depth of 313.4 meters below seafloor (mbsf). Two intervals making up about half the total penetration were cored (259.5-269.2 mbsf and 288.0-312.2 mbsf, Fig. 2), with an average recovery of $37 \%$. Essentially all the basalt recovered is nearly aphyric, containing less than $0.1 \%$ plagioclase phenocrysts. The core was divided on board ship into four units on the basis of crystallinity and the presence of glassy margins and xenoliths (Fig. 2). Chemically, the rocks range from N-MORB possibly to E-MORB with higher $\mathrm{TiO}_{2}$ and $\mathrm{K}_{2} \mathrm{O}$ contents, although the high $\mathrm{K}_{2} \mathrm{O}$ contents of the latter may 


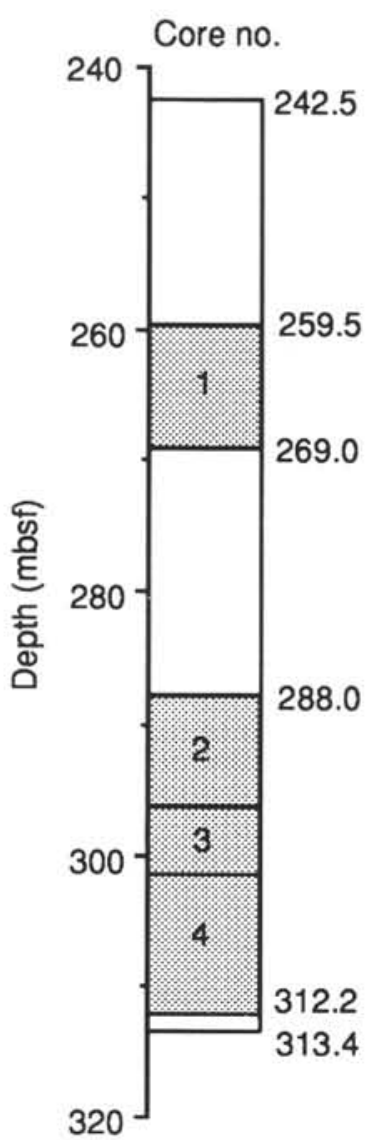

Figure 2. Lithostratigraphy of Hole $843 \mathrm{~B}$ basement. Each core (shaded) corresponds to a different lithologic unit.

be due in part to alteration effects (Dziewonski, Wilkens, Firth, et al., 1992). King et al. (this volume) provide detailed petrological and geochemical analyses of the rocks and conclude that they are mainly transitional MORB (T-MORB).

\section{METHODS}

Secondary minerals were identified by examination of polished thin sections, with compositional data from electron microprobe analyses. The latter were performed with a Cameca CAMEBAX instrument, using $15 \mathrm{kV}$ accelerating voltage, $10 \mathrm{nA}$ current, and rastering the beam over a square area, $6 \mu \mathrm{m}$ on a side. Whole-rock chemical analyses were performed by $\mathrm{X}$-ray fluorescence, with $\mathrm{CO}_{2}$ contents by coulometry, $\mathrm{H}_{2} \mathrm{O}$ by Karl Fischer titration, and ferrous iron by titration. Carbon and oxygen isotopic analyses of secondary carbonates separated from veins were performed on $\mathrm{CO}_{2}$ liberated by dissolution in phosphoric acid. Results are reported as $\delta$ notation in per mil relative to PDB and SMOW standards for carbon and oxygen, respectively.

\section{RESULTS}

\section{Phyllosilicate Mineralogy}

Phyllosilicates are the most abundant secondary phases, significantly influencing the chemistry of the rocks and providing important information on the conditions of alteration. Three basic types of phyllosilicate have been identified in basement from Hole 843B: celadonite, saponite, and Al-saponite. Structural formulas were calculated from chemical analyses on the basis of $\mathrm{O}_{10}(\mathrm{OH})_{2}$, and assuming all $\mathrm{Fe}$ as $\mathrm{Fe}^{3+}$ for celadonite and as $\mathrm{Fe}^{2+}$ for saponite (Table 1).

Celadonites from Hole 843B can be divided into two types: green celadonite is clearly optically distinguishable from blue-green cela-

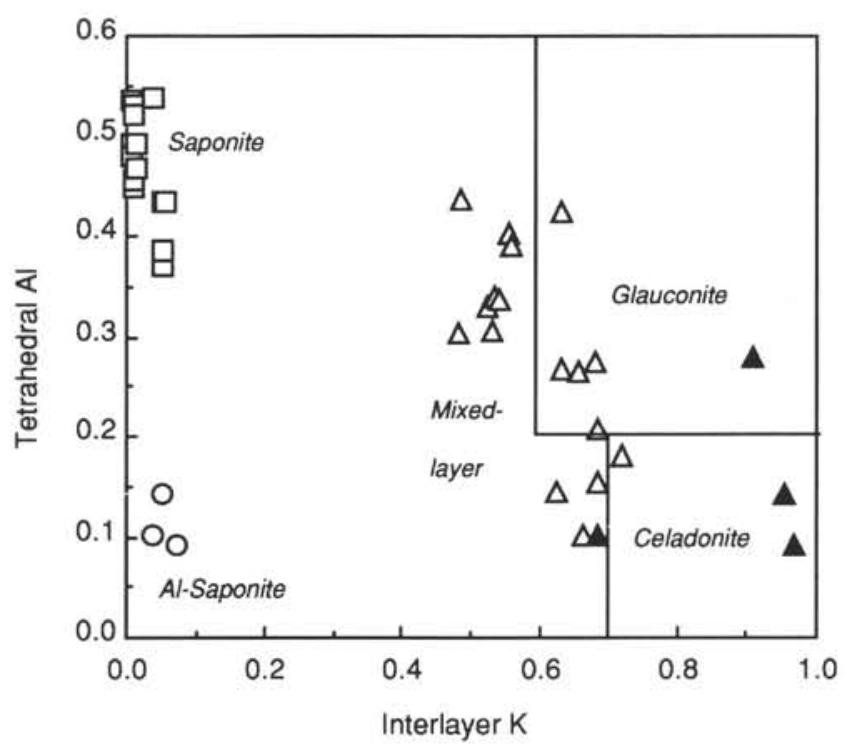

Figure 3. Tetrahedral Al vs. interlayer $\mathrm{K}$ contents from structural formulas of secondary phyllosilicates (moles per $\mathrm{O}_{10}(\mathrm{OH})_{2}$ ). Open squares = saponite; open circles = Al-saponite; open triangles = green celadonite; solid triangles = blue-green celadonite.

donite, which occurs later in the paragenesis. Both these types of "celadonite" actually range from true celadonite, with high $\mathrm{K}$ and low tetrahedral $\mathrm{Al}$ contents, to glauconite, which has higher tetrahedral $\mathrm{Al}$, and to mixed-layers or mixtures of celadonite or glauconite with smectite (Fig. 3). The trend in Figure 3 suggests that the smectite in these mixtures may be saponite (trioctahedral Mg-smectite), but all the celadonites are dioctahedral, with octahedral cation totals of about 2 (Fig. 4), suggesting that the smectite could be nontronite (dioctahedral $\mathrm{Fe}^{3+}$ smectite). Other analyses originally plotted along a trend between celadonite and saponite in Figure 3, but upon reexamination of the thin sections these analyses were found to be contaminated by adjacent phyllosilicates in zoned vesicle or vein fillings, so they are not reported.

Some consistent differences exist between the green and bluegreen celadonites. The green celadonites have lower $\mathrm{K}$ and higher $\mathrm{Al}$ contents and lower $\mathrm{Fe} / \mathrm{Fe}+\mathrm{Mg}$ ratios than the blue-green celadonites (Figs. 3 and 4). Yellowish to red celadonites also occur, but these are clearly mixtures of Fe-oxyhydroxides with celadonite, and have high $\mathrm{FeO}^{\mathrm{T}}$ contents and low $\mathrm{SiO}_{2}$ and $\mathrm{K}_{2} \mathrm{O}$ contents (e.g., analysis 34 in Table 1).

Saponites are brown to tan colored $\mathrm{Mg}$-rich smectites. They are trioctahedral, with low $\mathrm{Fe} / \mathrm{Fe}+\mathrm{Mg}$ ratios of $0.2-0.35$ (Figs. 3 and 4). $\mathrm{Ca}$ is the dominant interlayer cation.

Another brown to tan smectite is also rarely present as spherules and aggregates in calcite breccia cements and calcite veins. This phase has high $\mathrm{Al}$ contents $\left(0.7-1.1\right.$ atoms per formula $\left.\mathrm{O}_{10}(\mathrm{OH})_{2}, \mathrm{Fig} .4\right)$ and is mostly dioctahedral, with octahedral totals of $2.1-2.4$, and with $\mathrm{Ca}$ as the dominant interlayer cation. The origin of the layer charge is predominantly due to $\mathrm{Mg}^{2+}$ in octahedral positions, so it really should be classified as a montmorillonite transitional or intermediate to saponite, but due to its remarkable similarity to what has been termed Al-saponite in other altered seafloor basalts (Alt and Honnorez, 1984; Alt et al., 1992), it is referred to as Al-saponite in this paper.

\section{Alteration Types}

Three general alteration types can be recognized in the rocks on the basis of appearance in hand specimen and secondary mineralogy as determined in thin section. These include (1) dark halos around fractures and veins, (2) brown zones around fractures and veins, and (3) the gray host rocks. 

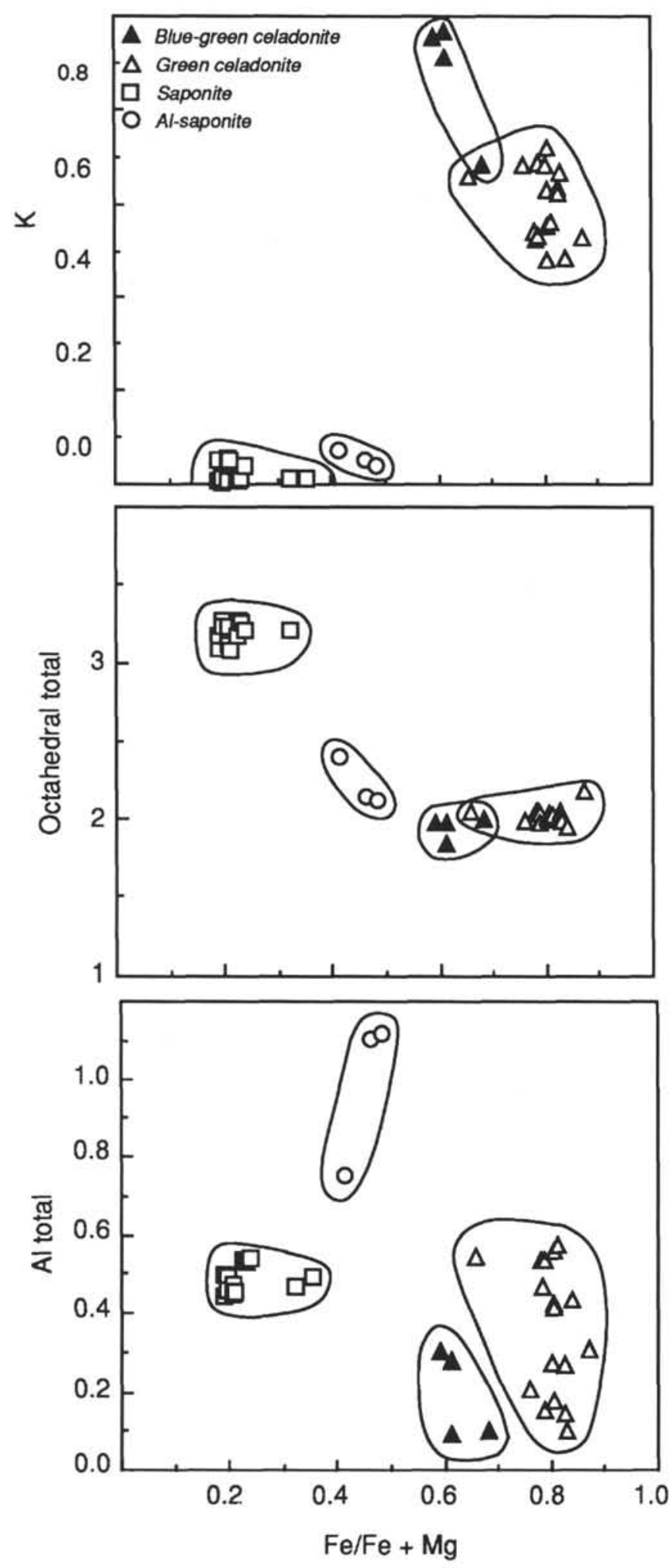

Figure 4. Compositions of secondary phyllosilicates. Units are moles per $\mathrm{O}_{10}(\mathrm{OH})_{2}$ of calculated structural formulas in Table 2.
Most of the core is characterized by the presence of $1-\mathrm{mm}$ - to 3 -cm-wide dark gray to black alteration halos (Fig. 5A) around veins filled with celadonite and Fe-oxyhydroxides \pm other phases (see "Veins" section below). In these halos, $5 \%-15 \%$ of the rock is altered, with pore space filled and olivine replaced by celadonite $\pm \mathrm{Fe}$-oxyhydroxides. Plagioclase and pyroxene are essentially unaltered. Titanomagnetite is generally slightly altered, exhibiting shrinkage cracks due to oxidation, but ranges to extensively corroded and altered, displaying internal reflections in reflected light. In some samples, vesicles and larger pores exhibit zoned fillings with the following sequences: (1) green celadonite, (2) Fe-oxyhydroxides, and (3) saponite; or (1) green celadonite, (2) blue-green celadonite, and (3) \pm calcite.

Brown zones, $1-3 \mathrm{~cm}$ wide, occur overlapping dark alteration halos along veins in Sections 136-843B-1R-3 and -1R-4, where veins are abundant (Fig. 5B). Narrow (1-3 mm) red zones also occur along some veins bordered by dark halos, and along veins in gray host rocks in Section 136-843B-3R-1. The rock in these brown and red zones is around $10 \%-15 \%$ altered, with $\mathrm{Fe}$-oxyhydroxides disseminated in the rock, filling pore spaces, and replacing olivine. Electron probe analyses of these $\mathrm{Fe}$-oxyhydroxides indicate high $\mathrm{FeO}^{\mathrm{T}}$ contents (up to $58 \%$ ), but significant contents of $\mathrm{K}_{2} \mathrm{O}$ and $\mathrm{SiO}_{2}$ also occur, suggesting that celadonite is present with the $\mathrm{Fe}$-oxyhydroxides (analyses 39 and 50 in Table 1).

The gray host rocks are about $15 \%$ altered, with pore spaces filled and olivine replaced by saponite \pm calcite. In some samples plagioclase and pyroxene are slightly altered to saponite. Titanomagnetite is slightly to extensively altered. Zoned vesicle fillings occur in some samples of gray host rock immediately adjacent to dark halos. In these cases the filling sequence is: (1) saponite, followed by (2) bluegreen celadonite.

\section{Veins}

Veins of phyllosilicates, Fe-oxyhydroxides, and calcite generally comprise about $1 \%-3 \%$ of the core, but are more abundant in two zones. Sections 136-843B-1R-3 and -1R-4, where broad brown alteration zones occur, contain $2 \%-5 \%$ veins (Fig. $5 \mathrm{~B}$ ), and Core 136-843B-2R contains about $10 \%$ veins and breccia cements of mainly calcite (Fig. 5C).

A general sequence of vein filling has been determined. (1) Green celadonite $\pm \mathrm{Fe}$-oxyhydroxides formed 0.05 - to 0.2 -mm-wide veins. (2) This was followed by staining of celadonite with $\mathrm{Fe}$-oxyhydroxides and precipitation of Fe-hydroxides in fractures. (3) Subsequently, either blue-green celadonite filled 0.5 - to $2-\mathrm{mm}$ fractures, saponite \pm pyrite formed $0.1-$ to $0.5-\mathrm{mm}$ veins, or calcite \pm pyrite \pm saponite filled $0.05-0.2 \mathrm{~mm}$ fractures. (4) Finally, late 0.2 - to 2 -mm-wide calcite \pm pyrite veins formed crosscutting earlier veins. Minor amounts of $0.05-$ to 0.1 -mm-wide veins of saponite alone \pm pyrite also occur in the rocks. In some cases, veins formed as sequentially zoned fracture fillings from green celadonite at the walls to Fe-oxyhydroxides to saponite at the center. In other cases, early green celadonite veins were refractured and cut by later blue-green celadonite and/or calcite veins. Calcite occurs both before and after blue-green celadonite in veins, and multiple generations of late crosscutting calcite veins are present in some samples.

Pyrite is most common in veins at the top of the core, whereas Fe-oxyhydroxides are particularly abundant in veins in Sections 136$843 \mathrm{~B}-1 \mathrm{R}-3$ and $-1 \mathrm{R}-4$, where vein frequency increases and where brown alteration zones are common. Blue-green celadonite \pm calcite veins are most abundant in Sections 136-843B-1R-1 and -2 and -2R-1. The calcite breccia cement in Section 136-843B-2R contains spherules and aggregates of $\mathrm{Al}$-saponite and $\mathrm{Fe}$-oxyhydroxides.

\section{Whole-Rock Chemistry}

Chemical analyses of samples for this study are listed in Table 2. The rocks exhibit high $\mathrm{H}_{2} \mathrm{O}^{+}$and $\mathrm{CO}_{2}$ contents $(0.6 \%-2.4 \%$ and $0.03 \%-1.42 \%$, respectively) due to hydration and calcite formation. 
Table 1. Electron microprobe analyses of phyllosilicates and calculated mineral formulas.

\begin{tabular}{|c|c|c|c|c|c|c|c|c|c|c|c|c|c|c|}
\hline $\begin{array}{l}\text { Analysis no. } \\
\text { Core-section } \\
\text { Interval (cm) } \\
\text { Mineral } \\
\text { Color } \\
\text { Occurrence }\end{array}$ & $\begin{array}{l}3 \\
\text { 1R-1 } \\
65-67 \\
\text { Celadonite } \\
\text { BG } \\
\text { Vein }\end{array}$ & $\begin{array}{l}6 \\
\text { IR-1 } \\
65-67 \\
\text { Celadonite } \\
\text { G } \\
\text { Vein }\end{array}$ & $\begin{array}{l}7 \\
\text { 1R-1 } \\
65-67 \\
\text { Saponite } \\
\text { Vein }\end{array}$ & $\begin{array}{l}\text { 13 } \\
\text { IR-1 } \\
65-67 \\
\text { Celadonite } \\
\text { G } \\
\text { Vug }\end{array}$ & $\begin{array}{l}14 \\
\text { IR-1 } \\
65-67 \\
\text { Celadonite } \\
\text { G } \\
\text { Vug }\end{array}$ & $\begin{array}{l}18 \\
18-1 \\
65-67 \\
\text { Saponite }\end{array}$ & $\begin{array}{l}19 \\
1 \mathrm{R}-2 \\
115-118 \\
\text { Celadonite } \\
\mathrm{G} \\
\text { Vein }\end{array}$ & $\begin{array}{l}22 \\
\text { 1R-2 } \\
115-118 \\
\text { Celadonite } \\
\text { BG } \\
\text { Vein }\end{array}$ & $\begin{array}{l}23 \\
\text { 1R-2 } \\
115-118 \\
\text { Celadonite } \\
\mathrm{G} \\
\text { Interstitial }\end{array}$ & $\begin{array}{l}25 \\
1 \text { R-3 } \\
52-54 \\
\text { Celadonite } \\
\text { G } \\
\text { Vug }\end{array}$ & $\begin{array}{l}26 \\
\text { IR-3 } \\
\text { 52-54 } \\
\text { Celadonite } \\
\text { G } \\
\text { Vug }\end{array}$ & $\begin{array}{l}\text { 31 } \\
\text { 1R-3 } \\
52-54 \\
\text { Celadonite } \\
\text { BG } \\
\text { Interstitial }\end{array}$ & $\begin{array}{l}32 \\
1 \mathrm{R}-3 \\
52-54 \\
\text { Celadonite } \\
\mathrm{G} \\
\text { Vug rim }\end{array}$ & $\begin{array}{l}33 \\
\text { IR-3 } \\
52-54 \\
\text { Celadonite } \\
\text { BG } \\
\text { Vug center }\end{array}$ \\
\hline \multicolumn{15}{|l|}{$\mathrm{Wt} \%$} \\
\hline $\mathrm{SiO}^{2}$ & 54.52 & 49.02 & 44.60 & 50.09 & 48.45 & 44.77 & 51.24 & 52.93 & 49.52 & 47.95 & 47.84 & 53.23 & 47.11 & 52.83 \\
\hline $\mathrm{Al}_{2} \mathrm{O}_{3}$ & 1.22 & 1.15 & 5.07 & 1.76 & 1.64 & 5.38 & 2.39 & 1.06 & 5.06 & 3.11 & 2.02 & 3.27 & 4.74 & 3.51 \\
\hline $\mathrm{MgO}$ & 6.01 & 3.48 & 18.73 & 4.04 & 3.40 & 17.62 & 4.49 & 7.15 & 2.83 & 3.80 & 3.79 & 6.56 & 3.55 & 6.79 \\
\hline $\mathrm{FeO}$ & 23.16 & 29.70 & 15.80 & 26.66 & 28.88 & 17.28 & 25.18 & 20.22 & 26.54 & 27.24 & 27.99 & 18.59 & 25.79 & 17.57 \\
\hline $\mathrm{MnO}$ & 0.05 & 0.02 & 0.04 & 0.03 & - & 0.07 & 0.08 & 0.06 & 0.06 & - & 0.01 & 0.07 & 0.07 & 0.02 \\
\hline $\mathrm{CaO}$ & 0.40 & & 0.85 & 0.54 & 0.47 & 1.45 & 0.45 & 0.05 & 0.74 & 0.47 & 0.40 & 0.14 & 0.60 & 0.09 \\
\hline $\mathrm{Na}_{2} \mathrm{O}$ & 0.03 & 0.04 & 0.15 & 0.07 & 0.09 & 0.13 & 0.04 & 0.03 & 0.46 & 0.12 & 0.06 & 0.03 & 0.05 & 0.01 \\
\hline $\mathrm{K}_{2} \mathrm{O}$ & 7.54 & 7.00 & 0.14 & 7.23 & 6.49 & 0.13 & 7.33 & 10.37 & 5.20 & 7.12 & 7.46 & 9.85 & 6.54 & 10.26 \\
\hline $\mathrm{TiO}_{2}$ & 0.03 & 0.00 & - & 0.01 & 0.08 & 0.04 & 0.02 & 0.03 & 0.41 & 0.03 & 0.04 & 0.07 & 0.16 & 0.06 \\
\hline Total & 92.93 & 90.91 & 85.37 & 90.43 & 89.50 & 86.88 & 91.23 & 91.90 & 90.83 & 89.83 & 89.60 & 91.82 & 88.61 & 91.14 \\
\hline \multicolumn{15}{|c|}{$\begin{array}{l}\text { Calculated formula } \\
\text { atoms/11 oxygens } \\
\text { Tetrahedral }\end{array}$} \\
\hline $\mathrm{Si}$ & 3.883 & 3.656 & 3.494 & 3.724 & 3.656 & 3.479 & 3.753 & 3.870 & 3.624 & 3.605 & 3.624 & 3.856 & 3.569 & 3.857 \\
\hline $\mathrm{Fe}^{3+}$ & 0.015 & 0.243 & & 0.122 & 0.199 & & 0.041 & 0.039 & 0.000 & 0.120 & 0.195 & 0.000 & 0.008 & 0.000 \\
\hline \multirow{2}{*}{\multicolumn{15}{|c|}{ Octahedral }} \\
\hline & & & & & & & & & & & & & & \\
\hline $\mathrm{Mg}$ & 0.638 & 0.387 & 2.186 & 0.447 & 0.382 & 2.040 & 0.490 & 0.779 & 0.308 & 0.426 & 0.428 & $\begin{array}{l}0.1308 \\
0.708\end{array}$ & 0.401 & 0.738 \\
\hline $\mathrm{Fe}^{3+}$ & 1.361 & 1.605 & 0.000 & 1.532 & 1.619 & 0.000 & 1.498 & 1.194 & 1.620 & 1.588 & 1.573 & 1.123 & 1.622 & 1.070 \\
\hline $\mathrm{Fe}^{2+}$ & 0.000 & 0.000 & 1.032 & 0.000 & 0.000 & 1.120 & 0.000 & 0.000 & 0.000 & 0.000 & 0.000 & 0.000 & 0.000 & 0.000 \\
\hline Mn & 0.003 & 0.001 & 0.003 & 0.002 & 0.000 & 0.005 & 0.005 & 0.004 & 0.004 & 0.000 & 0.001 & 0.004 & 0.004 & 0.001 \\
\hline $\mathrm{Ti}$ & 0.002 & 0.000 & 0.000 & 0.001 & 0.005 & 0.002 & 0.001 & 0.002 & 0.023 & 0.002 & 0.002 & 0.004 & 0.009 & 0.003 \\
\hline Octahedral Total & 2.003 & 1.993 & 3.221 & 1.981 & 2.006 & 3.167 & 1.994 & 1.978 & 2.015 & 2.016 & 2.004 & 1.975 & 2.036 & 1.972 \\
\hline \multicolumn{15}{|l|}{ Interlayer } \\
\hline & 0.031 & 0.041 & 0.071 & 0.043 & 0.038 & 0.121 & 0.035 & 0.004 & 0.058 & 0.038 & 0.033 & 0.011 & 0.049 & 0.007 \\
\hline $\mathrm{Na}$ & 0.004 & 0.006 & 0.023 & 0.010 & 0.013 & 0.019 & 0.006 & 0.004 & 0.065 & 0.017 & 0.009 & 0.004 & 0.007 & 0.001 \\
\hline K & 0.685 & 0.666 & 0.014 & 0.685 & 0.624 & 0.013 & 0.685 & 0.967 & 0.485 & 0.683 & 0.721 & 0.910 & 0.632 & 0.955 \\
\hline Interlayer total & 0.719 & 0.712 & 0.108 & 0.739 & 0.676 & 0.153 & 0.726 & 0.975 & 0.608 & 0.738 & 0.762 & 0.925 & 0.688 & 0.964 \\
\hline Al Total & 0.102 & 0.101 & 0.468 & 0.154 & 0.146 & 0.492 & 0.206 & 0.091 & 0.436 & 0.275 & 0.180 & 0.279 & 0.423 & 0.302 \\
\hline $\mathrm{Fe}^{\mathrm{T}}$ & 1.376 & 1.848 & 1.032 & 1.653 & 1.818 & 1.120 & 1.539 & 1.233 & 1.620 & 1.708 & 1.769 & 1.123 & 1.630 & 1.070 \\
\hline $\mathrm{Fe} / \mathrm{Fe}+\mathrm{Mg}$ & 0.683 & 0.827 & 0.321 & 0.787 & 0.826 & 0.355 & 0.758 & 0.613 & 0.840 & 0.801 & 0.805 & 0.613 & 0.803 & 0.592 \\
\hline
\end{tabular}


Table 1 (continued).

\begin{tabular}{|c|c|c|c|c|c|c|c|c|c|c|c|c|c|c|}
\hline $\begin{array}{l}\text { Analysis no. } \\
\text { Core-section } \\
\text { Interval (cm) } \\
\text { Mineral } \\
\text { Color } \\
\text { Occurrence }\end{array}$ & $\begin{array}{l}34 \\
1 \mathrm{R}-3 \\
52-54 \\
\text { Celadonite } \\
\text { Red } \\
\text { Olivine }\end{array}$ & $\begin{array}{l}35 \\
\text { 1R-5 } \\
36-38 \\
\text { Celadonite } \\
\text { G } \\
\text { Vug }\end{array}$ & $\begin{array}{l}36 \\
\text { IR-5 } \\
36-38 \\
\text { Saponite } \\
\text { Vug }\end{array}$ & $\begin{array}{l}37 \\
\text { 1R-5 } \\
36-38 \\
\text { Celadonite } \\
\text { G } \\
\text { Vug rim }\end{array}$ & $\begin{array}{l}39 \\
1 \mathrm{R}-5 \\
36-38 \\
\text { Fe-oxide }\end{array}$ & $\begin{array}{l}40 \\
\text { 1R-5 } \\
36-38 \\
\text { Saponite }\end{array}$ & $\begin{array}{l}42 \\
\text { IR-5 } \\
36-38 \\
\text { Saponite }\end{array}$ & $\begin{array}{l}43 \\
\text { 1R-5 } \\
\text { 36-38 } \\
\text { Saponite }\end{array}$ & $\begin{array}{l}44 \\
4 \mathrm{R}-1 \\
12-15 \\
\text { Saponite }\end{array}$ & $\begin{array}{l}46 \\
4 \mathrm{R}-1 \\
12-15 \\
\text { Saponite }\end{array}$ & $\begin{array}{l}47 \\
4 \mathrm{R}-1 \\
12-15 \\
\text { Celadonite } \\
\mathrm{G} \\
\text { Vug }\end{array}$ & $\begin{array}{l}48 \\
4 \mathrm{R}-1 \\
12-15 \\
\text { Celadonite } \\
\mathrm{G} \\
\text { Vug }\end{array}$ & $\begin{array}{l}49 \\
4 R-1 \\
12-15 \\
\text { Celadonite } \\
\text { G } \\
\text { Vug }\end{array}$ & $\begin{array}{l}50 \\
4 \mathrm{R}-1 \\
12-15 \\
\text { Fe-oxide } \\
\text { Vug }\end{array}$ \\
\hline \multicolumn{15}{|l|}{$\mathrm{W}_{1} \%$} \\
\hline $\mathrm{SiO}_{2}$ & 39.42 & 49.85 & 46.50 & 46.61 & 21.77 & 47.70 & 46.48 & 46.31 & 44.56 & 44.13 & 49.45 & 49.30 & 48.83 & 16.76 \\
\hline $\mathrm{Al}_{2} \mathrm{O}_{3}$ & 3.24 & 4.77 & 4.83 & 2.97 & 1.72 & 5.06 & 5.21 & 5.21 & 5.35 & 5.30 & 6.11 & 6.14 & 5.26 & 2.09 \\
\hline $\mathrm{MgO}$ & 2.92 & 3.44 & 21.28 & 3.41 & 2.46 & 20.91 & 21.40 & 21.34 & 22.64 & 21.55 & 3.67 & 3.55 & 3.70 & 1.22 \\
\hline $\mathrm{FeO}$ & 34.87 & 25.01 & 8.86 & 28.42 & 52.58 & 9.88 & 9.98 & 9.93 & 9.90 & 9.04 & 23.31 & 23.38 & 23.87 & 57.79 \\
\hline $\mathrm{MnO}$ & 0.08 & 0.04 & 0.01 & & 0.18 & 0.07 & 0.05 & 0.03 & 0.05 & 0.05 & 0.07 & 0.04 & 0.06 & 0.18 \\
\hline $\mathrm{CaO}$ & 0.68 & 0.44 & 0.50 & 0.39 & 0.46 & 0.31 & 0.53 & 0.45 & 0.39 & 0.50 & 0.43 & 0.49 & 0.46 & 0.52 \\
\hline $\mathrm{Na}_{2} \mathrm{O}$ & 0.10 & 0.08 & 0.02 & 0.05 & - & 0.08 & 0.05 & 0.07 & 0.01 & 0.04 & & . & & - \\
\hline $\mathrm{K}_{2} \mathrm{O}$ & 5.19 & 5.09 & 0.53 & 6.49 & 2.73 & 0.53 & 0.54 & 0.56 & 0.06 & 0.07 & 5.73 & 5.64 & 5.47 & 1.82 \\
\hline $\mathrm{TiO}_{2}$ & 0.21 & 0.03 & 0.05 & 0.19 & 0.62 & 0.03 & 0.03 & 0.02 & 0.04 & 0.01 & 0.02 & 0.02 & 0.03 & 0.22 \\
\hline Total & 86.71 & 88.75 & 82.57 & 88.53 & 82.53 & 84.57 & 84.25 & 83.93 & 82.99 & 80.68 & 88.79 & 88.55 & 87.68 & 80.60 \\
\hline \multicolumn{15}{|l|}{$\begin{array}{l}\text { Calculated } \\
\text { formula(atoms) } \\
\text { Tetrahedral }\end{array}$} \\
\hline $\mathrm{Si}$ & 3.167 & 3.695 & 3.613 & 3.564 & & 3.630 & 3.566 & 3.566 & 3.474 & 3.519 & 3.663 & 3.660 & 3.669 & \\
\hline $\mathrm{Fe}^{3+}$ & 0.526 & 0.000 & 0.000 & 0.168 & & 0.000 & 0.000 & 0.000 & - & 0.000 & 0.000 & 0.000 & 0.000 & \\
\hline $\begin{array}{l}\text { Al } \\
\text { Octahedral }\end{array}$ & 0.307 & 0.305 & 0.387 & 0.267 & & 0.370 & 0.434 & 0.434 & 0.491 & 0.481 & 0.337 & 0.340 & 0.331 & \\
\hline $\mathrm{Al}$ & 0.000 & 0.112 & 0.055 & 0.000 & & 0.083 & 0.036 & 0.039 & 0.000 & 0.017 & 0.196 & 0.197 & 0.135 & \\
\hline $\mathrm{Mg}$ & 0.349 & 0.380 & 2.463 & 0.388 & & 2.370 & 2.445 & 2.448 & 2.629 & 2.560 & 0.405 & 0.393 & 0.414 & \\
\hline $\mathrm{Fe}^{3+}$ & 1.811 & 1.546 & 0.000 & 1.644 & & 0.000 & 0.000 & 0.000 & 0.000 & 0.000 & 1.440 & 1.448 & 1.496 & \\
\hline $\mathrm{Fe}^{2+}$ & 0.000 & 0.000 & 0.574 & 0.000 & & 0.627 & 0.639 & 0.638 & 0.644 & 0.601 & 0.000 & 0.000 & 0.000 & \\
\hline Mn & 0.005 & 0.003 & 0.001 & 0.000 & & 0.005 & 0.003 & 0.002 & 0.003 & 0.003 & 0.004 & 0.003 & 0.004 & \\
\hline $\mathrm{Ti}$ & 0.013 & 0.002 & 0.003 & 0.011 & & 0.002 & 0.002 & 0.001 & 0.002 & 0.001 & 0.001 & 0.001 & 0.002 & \\
\hline Octahedral Total & 2.179 & 2.042 & 3.096 & 2.044 & & 3.087 & 3.125 & 3.128 & 3.279 & 3.182 & 2.046 & 2.041 & 2.051 & \\
\hline \multicolumn{15}{|l|}{ Interlayer } \\
\hline $\mathrm{Ca}$ & 0.059 & 0.035 & 0.042 & 0.032 & & 0.025 & 0.044 & 0.037 & 0.033 & 0.043 & 0.034 & 0.039 & 0.037 & \\
\hline $\mathrm{Na}$ & 0.016 & 0.011 & 0.003 & 0.007 & & 0.012 & 0.007 & 0.010 & 0.002 & 0.006 & 0.000 & 0.000 & 0.000 & \\
\hline K & 0.532 & 0.481 & 0.053 & 0.633 & & 0.051 & 0.053 & 0.055 & 0.006 & 0.007 & 0.541 & 0.534 & 0.524 & \\
\hline Interlayer total & 0.606 & 0.528 & 0.097 & 0.672 & & 0.088 & 0.104 & 0.103 & 0.040 & 0.056 & 0.575 & 0.573 & 0.561 & \\
\hline $\begin{array}{l}\text { Al Total } \\
\text { FoT }\end{array}$ & 0.307 & 0.416 & 0.442 & 0.267 & & 0.453 & 0.471 & 0.472 & 0.491 & 0.498 & 0.533 & 0.537 & 0.465 & \\
\hline $\mathrm{Fe}^{\mathrm{T}}$ & 2.337 & 1.546 & 0.574 & 1.813 & & 0.627 & 0.639 & 0.638 & 0.644 & 0.601 & 1.440 & 1.448 & 1.496 & \\
\hline $\mathrm{Fe} / \mathrm{Fe}+\mathrm{Mg}$ & 0.870 & 0.803 & 0.189 & 0.824 & & 0.209 & 0.207 & 0.207 & 0.197 & 0.190 & 0.781 & 0.787 & 0.783 & \\
\hline
\end{tabular}


Table 1 (continued).

\begin{tabular}{|c|c|c|c|c|c|c|c|c|c|c|c|c|}
\hline $\begin{array}{l}\text { Analysis no. } \\
\text { Core-section } \\
\text { Interval (cm) } \\
\text { Mineral } \\
\text { Color } \\
\text { Occurrence }\end{array}$ & $\begin{array}{l}51 \\
3 R-1 \\
17-19 \\
\text { Saponite }\end{array}$ & $\begin{array}{l}52 \\
3 \mathrm{R}-1 \\
17-19 \\
\text { Saponite }\end{array}$ & $\begin{array}{l}53 \\
3 \mathrm{R}-1 \\
17-19 \\
\text { Saponite }\end{array}$ & $\begin{array}{l}54 \\
3 \mathrm{R}-1 \\
17-19 \\
\text { Celadonite } \\
\text { Red } \\
\text { Vug }\end{array}$ & $\begin{array}{l}55 \\
1 \mathrm{R}-6 \\
7-9 \\
\text { Celadonite } \\
\mathrm{G} \\
\text { Vug }\end{array}$ & $\begin{array}{l}56 \\
1 \mathrm{R}-6 \\
7-9 \\
\text { Celadonite } \\
\mathrm{G} \\
\text { Vug }\end{array}$ & $\begin{array}{l}57 \\
1 R-6 \\
7-9 \\
\text { Saponite }\end{array}$ & $\begin{array}{l}58 \\
1 \mathrm{R}-6 \\
7-9 \\
\text { Saponite }\end{array}$ & $\begin{array}{l}59 \\
\text { IR-6 } \\
7-9 \\
\text { Saponite }\end{array}$ & $\begin{array}{l}60 \\
2 R-2 \\
38-40 \\
\text { Al-saponite }\end{array}$ & $\begin{array}{l}61 \\
2 \mathrm{R}-2 \\
38-40 \\
\text { Al-saponite } \\
\text { Breccia }\end{array}$ & $\begin{array}{l}62 \\
2 \mathrm{R}-2 \\
38-40 \\
\text { Al-saponite }\end{array}$ \\
\hline \multicolumn{13}{|l|}{$W_{t} \%$} \\
\hline $\mathrm{SiO}_{2}$ & 42.41 & 42.72 & 43.55 & 50.16 & 48.58 & 48.28 & 45.04 & 43.62 & 45.45 & 55.45 & 43.13 & 54.83 \\
\hline $\mathrm{Al}_{2} \mathrm{O}_{3}$ & 5.58 & 5.66 & 5.76 & 6.19 & 6.42 & 6.53 & 4.87 & 5.70 & 5.01 & 13.64 & 7.94 & 13.53 \\
\hline $\mathrm{MgO}$ & 20.95 & 21.24 & 20.74 & 5.45 & 3.33 & 3.12 & 22.18 & 20.64 & 22.54 & 5.64 & 10.59 & 6.46 \\
\hline $\mathrm{FeO}$ & 11.16 & 11.07 & 11.49 & 18.69 & 24.35 & 23.98 & 10.19 & 10.63 & 9.90 & 9.41 & 13.20 & 9.85 \\
\hline $\mathrm{MnO}$ & 0.06 & 0.06 & 0.03 & & 0.05 & 0.01 & 0.06 & 0.10 & 0.06 & 0.02 & 0.09 & \\
\hline $\mathrm{CaO}$ & 1.11 & 0.69 & 0.39 & 0.29 & 0.64 & 0.48 & 0.54 & 0.61 & 0.52 & 1.86 & 1.98 & 2.26 \\
\hline $\mathrm{Na}_{2} \mathrm{O}$ & 0.13 & 0.13 & 0.13 & 0.19 & 0.09 & 0.11 & 0.12 & 0.15 & 0.10 & 0.28 & 1.31 & 0.46 \\
\hline $\mathrm{K}_{2} \mathrm{O}$ & 0.10 & 0.07 & 0.39 & 6.92 & 5.88 & 5.88 & 0.10 & 0.12 & 0.11 & 0.43 & 0.70 & 0.59 \\
\hline $\mathrm{TiO}_{2}$ & & & & 0.05 & 0.11 & 0.05 & 0.01 & 0.04 & 0.02 & 0.04 & 0.09 & 0.06 \\
\hline Total & 81.49 & 81.64 & 82.47 & 87.92 & 89.44 & 88.45 & 83.10 & 81.62 & 83.72 & 86.77 & 79.03 & 88.03 \\
\hline \multicolumn{13}{|l|}{$\begin{array}{l}\text { Calculated } \\
\text { formula(atoms) } \\
\text { Tetrahedral }\end{array}$} \\
\hline $\mathrm{Si}$ & 3.415 & 3.423 & 3.457 & 3.736 & 3.597 & 3.609 & 3.513 & 3.479 & 3.512 & 3.866 & 3.474 & 3.798 \\
\hline $\mathrm{Fe}^{3+}$ & 0.000 & 0.000 & 0.000 & 0.000 & 0.000 & 0.000 & 0.000 & 0.000 & 0.000 & 0.000 & 0.000 & 0.000 \\
\hline Al & 0.529 & 0.534 & 0.538 & 0.264 & 0.403 & 0.391 & 0.447 & 0.521 & 0.456 & 0.134 & 0.526 & 0.202 \\
\hline $\begin{array}{l}\text { Al } \\
\mathrm{Al}\end{array}$ & 0.000 & 0.000 & 0.000 & 0.279 & 0.156 & 0.184 & 0.000 & 0.015 & 0.000 & 0.986 & 0.228 & 0.902 \\
\hline $\mathrm{Mg}$ & 2.513 & 2.535 & 2.452 & 0.605 & 0.367 & 0.347 & 2.577 & 2.452 & 2.594 & 0.586 & 1.271 & 0.667 \\
\hline $\mathrm{Fe}^{+3}$ & 0.000 & 0.000 & 0.000 & 1.161 & 1.504 & 1.495 & 0.000 & 0.000 & 0.000 & 0.547 & 0.887 & 0.569 \\
\hline $\mathrm{Fe}^{2+}$ & 0.750 & 0.740 & 0.761 & 0.000 & 0.000 & 0.000 & 0.663 & 0.707 & 0.638 & 0.000 & 0.000 & 0.000 \\
\hline $\mathrm{Mn}$ & 0.004 & 0.004 & 0.002 & 0.000 & 0.003 & 0.001 & 0.004 & 0.007 & 0.004 & 0.001 & 0.006 & 0.000 \\
\hline $\mathrm{Ti}$ & 0.000 & 0.000 & 0.000 & 0.003 & 0.006 & 0.003 & 0.001 & 0.002 & 0.001 & 0.002 & 0.005 & 0.003 \\
\hline Octahedral Total & 3.266 & 3.279 & 3.215 & 2.047 & 2.037 & 2.030 & 3.244 & 3.183 & 3.237 & 2.123 & 2.397 & 2.141 \\
\hline \multicolumn{13}{|l|}{ Interlaver } \\
\hline & 0.096 & 0.059 & 0.033 & 0.023 & 0.051 & 0.038 & 0.045 & 0.052 & 0.043 & 0.139 & 0.171 & 0.168 \\
\hline $\mathrm{Na}$ & 0.020 & 0.020 & 0.020 & 0.027 & 0.013 & 0.016 & 0.018 & 0.023 & 0.015 & & 0.204 & 0.061 \\
\hline $\mathrm{K}$ & 0.010 & 0.007 & 0.039 & 0.657 & 0.555 & 0.560 & 0.010 & 0.012 & 0.011 & 0.038 & 0.072 & 0.052 \\
\hline Interlayer total & 0.126 & 0.087 & 0.093 & 0.708 & 0.619 & 0.615 & 0.073 & 0.087 & 0.069 & 0.215 & 0.447 & 0.281 \\
\hline Al Total & 0.529 & 0.534 & 0.538 & 0.543 & 0.560 & 0.575 & 0.447 & 0.535 & 0.456 & 1.120 & 0.753 & 1.104 \\
\hline $\mathrm{Fe}^{\mathrm{T}}$ & 0.750 & 0.740 & 0.761 & 1.161 & 1.504 & 1.495 & 0.663 & 0.707 & 0.638 & 0.547 & 0.887 & 0.569 \\
\hline $\mathrm{Fe} / \mathrm{Fe}+\mathrm{Mg}$ & 0.230 & 0.226 & 0.237 & 0.658 & 0.804 & 0.811 & 0.205 & 0.224 & 0.197 & 0.483 & 0.411 & 0.461 \\
\hline
\end{tabular}

Notes: $\mathrm{G}$ = green; $\mathrm{BG}$ = blue-green; dashes = none detected; blanks = not analyzed. 
A

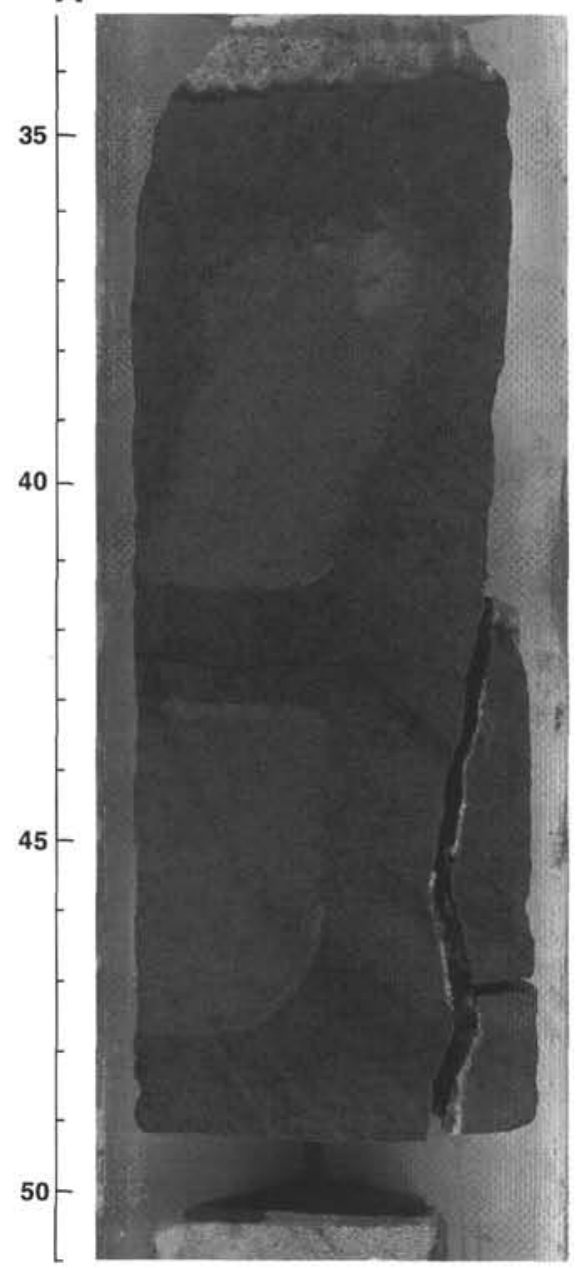

B

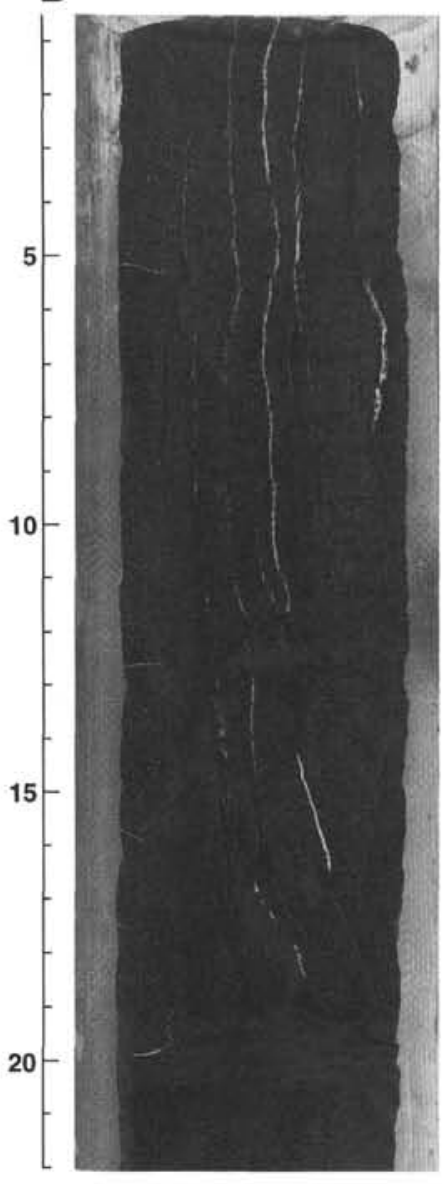

C

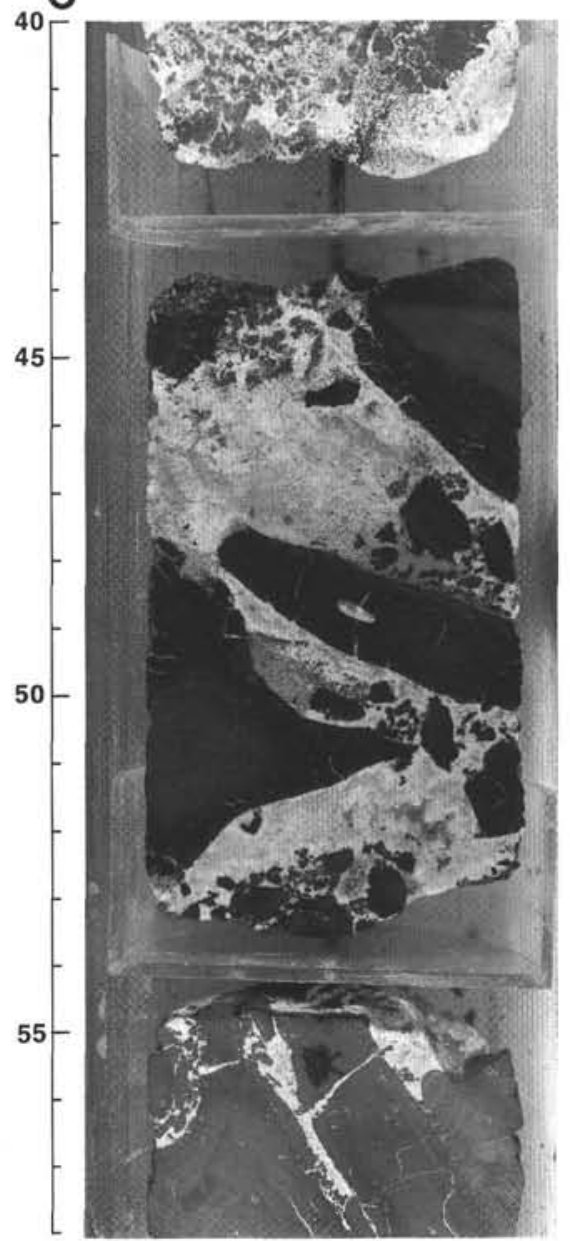

Figure 5. Photographs of altered rocks from Hole 843B. A. Typical dark halo alteration zones around celadonite \pm later calcite veins (at center, bottom, and right side of core) and adjacent gray host rock in Core $843 \mathrm{~B}-1 \mathrm{R}-2$. B. Abundant vertical veins of celadonite, Fe-oxyhydroxides, and late calcite surrounded by reddish-brown altered rock and gray host rock farther from the veins in Core $843 \mathrm{~B}-1 \mathrm{R}-4$. C. Brecciated rock cemented by calcite in Core $843 \mathrm{~B}-2 \mathrm{R}-2$.

Because the main alteration phases are hydrated phyllosilicates and oxides plus calcite, the amount of volatiles in the rocks is a good indicator of extent of alteration. To eliminate the effects of dilution of other elements caused by addition of $\mathrm{H}_{2} \mathrm{O}$ and $\mathrm{CO}_{2}$ to the rocks, the analyses were recalculated volatile-free and plotted vs. loss on ignition in Figure 6. Relative to the host gray rocks, the dark halos and brown zones have lower $\mathrm{SiO}_{2}, \mathrm{Al}_{2} \mathrm{O}_{3}, \mathrm{MgO}$, and $\mathrm{TiO}_{2}$ contents, exhibit elevated $\mathrm{Fe}_{2} \mathrm{O}_{3}{ }^{\mathrm{T}}, \mathrm{K}_{2} \mathrm{O}, \mathrm{MnO}, \mathrm{H}_{2} \mathrm{O}^{+}$, and $\mathrm{CO}_{2}$ contents, and have higher loss on ignition and $\mathrm{Fe}^{3+} / \mathrm{Fe}^{\mathrm{T}}$ (Table 2, Fig. 6). $\mathrm{Na}_{2} \mathrm{O}$ contents generally decrease in the dark halos, but increase in one sample (843B-2R-2, 38-40 $\mathrm{cm}$ ), and $\mathrm{CaO}$ shows no consistent changes. There are possible increases in $\mathrm{Ba}$ and decreases in $\mathrm{Ni}$ and $\mathrm{Sr}$ contents from gray host rocks to dark halos and brown zones, but these are small and somewhat variable (Table 2). Chemical changes in the brown zones are similar to those in the dark halos, but the brown zones tend to exhibit greater changes and have the highest $\mathrm{Fe}_{2} \mathrm{O}_{3}{ }^{\mathrm{T}}$ and $\mathrm{H}_{2} \mathrm{O}^{+}$contents and $\mathrm{Fe}^{3+} / \mathrm{Fe}^{\mathrm{T}}$ ratios (Fig. 6).

\section{Stable Isotopic Compositions of Vein Carbonates}

Isotopic compositions of vein carbonates are given in Table $3 . \delta^{13} \mathrm{C}$ values are fairly uniform, ranging from $+1.4 \%$ to $+2.8 \%$, and $\delta^{18} \mathrm{O}$ values range from $+25.5 \%$ to $+30.5 \%$. The $\delta^{13} \mathrm{C}$ values of calcites are consistent with their formation from fluids with carbon isotopic compositions of normal seawater. Assuming formation in equilibrium with fluid of normal seawater $\delta^{18} \mathrm{O}$ value $(0 \%)$, the calcites formed at temperatures ranging from about $10^{\circ}$ to $40^{\circ} \mathrm{C}$ (Table 3; O'Neil et al., 1969). If the calcites formed from Cretaceous seawater $\left(\delta^{18} \mathrm{O}=-1 \%\right.$; Shackleton and Kennett, 1975) or from basement fluids that were slightly depleted in ${ }^{18} \mathrm{O}$, like deep-sea sediment pore waters (Lawrence and Gieskes, 1981), then temperatures were somewhat lower: assuming a fluid $\delta^{18} \mathrm{O}$ of $-2 \%$ gives temperatures of about $5^{\circ}$ to $30^{\circ} \mathrm{C}$.

\section{DISCUSSION}

The secondary mineralogy and the distribution of alteration zones around veins in Hole 843B are typical of oceanic volcanics affected by low temperature $\left(0^{\circ}-100^{\circ} \mathrm{C}\right.$ ) interaction with seawater (Andrews, 1977; Laverne and Vivier, 1983; Alt and Honnorez, 1984; Böhlke et al., 1984; Alt, Honnorez, Laverne, and Emmermann, 1986). The secondary mineralogy and sequences of mineral formation in veins, plus comparison of Hole $843 \mathrm{~B}$ rocks with older and younger seafloor rocks, allow constraints to be placed on the timing and conditions of 

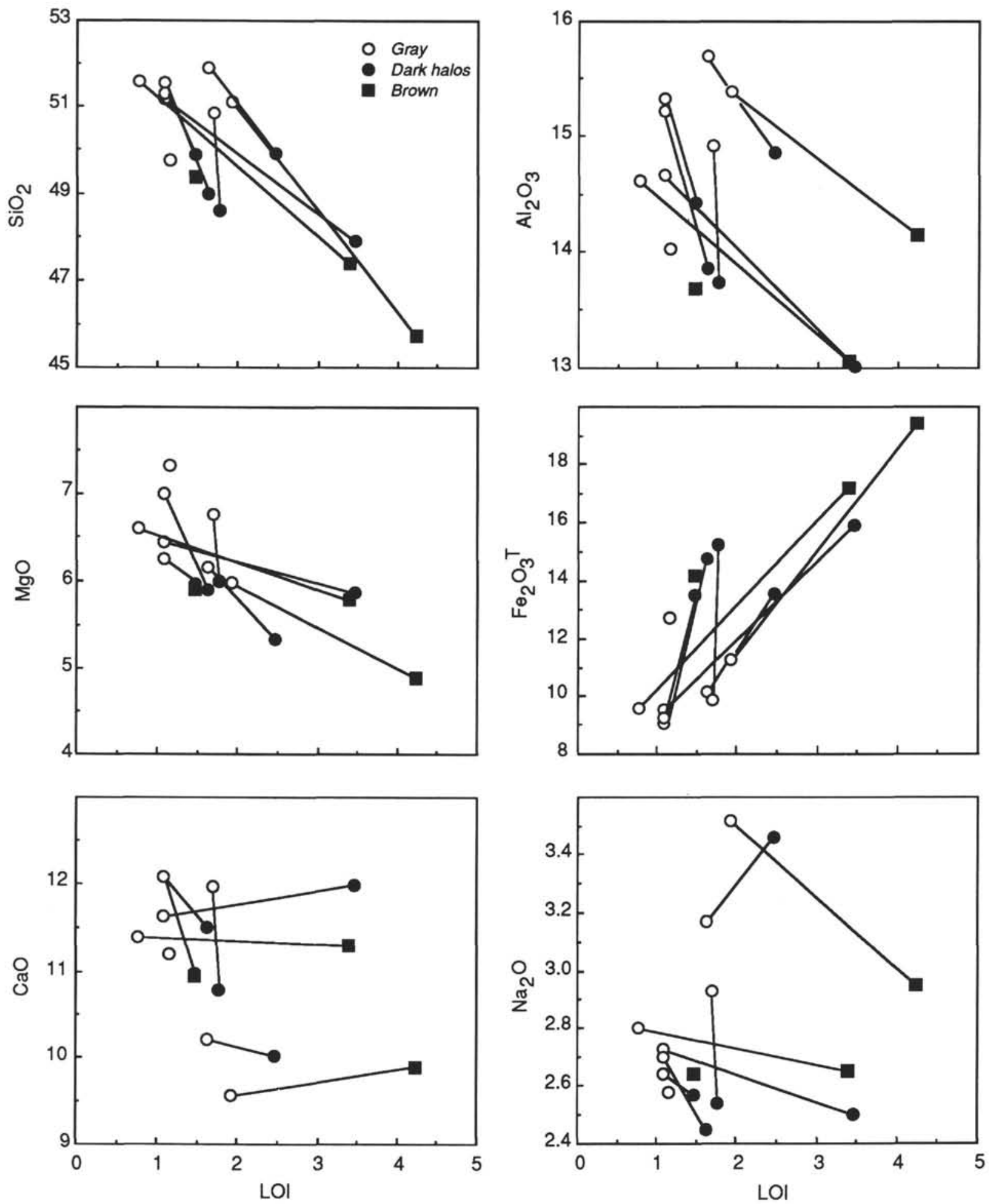

Figure 6. Chemical compositions of Hole 843B basalts plotted vs. LOI (loss on ignition). Analyses (except LOI) normalized volatile-free. Lines connect analyses of alteration halo and adjacent host rock.

alteration at Site 843B. The general sequence recorded in Hole 843B is the following: (1) green celadonite $\pm \mathrm{Fe}$-oxyhydroxides; (2) Feoxyhydroxides; (3) either (a) blue-green celadonite, (b) saponite \pm pyrite, or (c) calcite \pm pyrite; and finally (4) late calcite \pm pyrite.

Celadonite contains predominantly ferric iron (Alt and Honnorez, 1984; Table 2) and is associated with ferric oxyhydroxides, indicating oxidizing conditions and suggesting relatively open circulation of seawater during its formation. The increased oxidation of iron in the dark halos is consistent with alteration and celadonite formation under oxidizing conditions. The iron in celadonite may have been derived locally by expulsion from titanomagnetite during its oxidation (Petersen et al., 1979), but the increase in total iron in the dark halos suggests that $\mathrm{Fe}$ was derived externally to the rock. The configuration of dark halos around fractures and veins indicates that the Fe entered the rock 

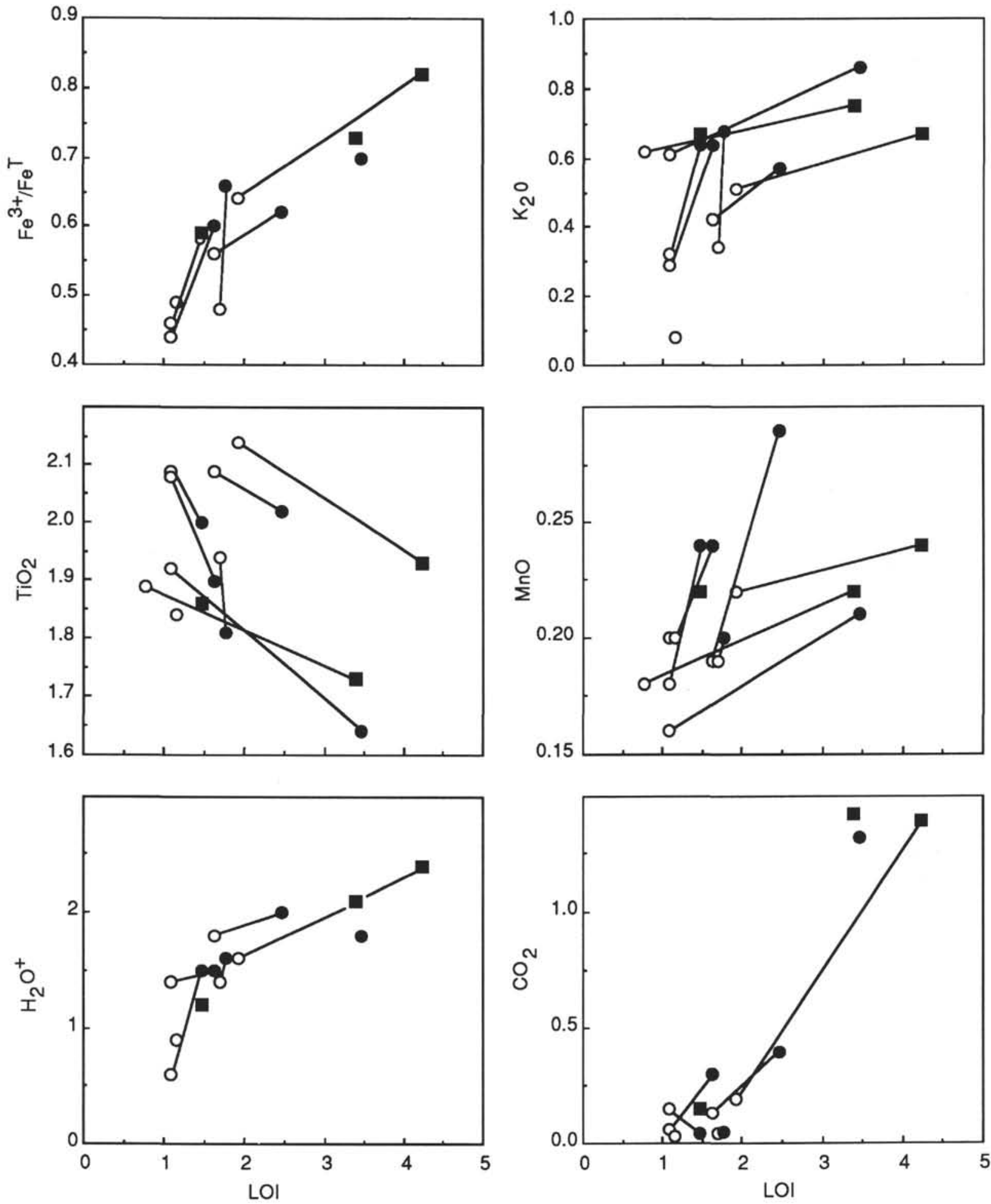

Figure 6 (continued).

from circulating Fe-rich solutions. Iron in solution may have been derived from breakdown of volcanic glass at local pillow and flow margins, or transported from reactions deeper in the volcanic section, possibly at higher temperature. The latter seems more likely given that more reducing alteration reactions prevail deeper in oceanic volcanics (Alt and Honnorez, 1984; Alt, Honnorez, Laverne, and Emmermann, 1986), and such solutions would readily transport reduced iron in solution. The celadonite in Hole 843B rocks probably formed at temperatures similar to those at which it formed in other altered seafloor basalts, less than about $50^{\circ} \mathrm{C}$ (Kastner and Gieskes, 1976; Seyfried et al., 1978; Stakes and O'Neil, 1982; Böhlke et al., 1984).

The losses of $\mathrm{SiO}_{2}$ and $\mathrm{MgO}$ and increases in $\mathrm{Fe}_{2} \mathrm{O}_{3}{ }^{\mathrm{T}}, \mathrm{K}_{2} \mathrm{O}, \mathrm{H}_{2} \mathrm{O}^{+}$, and $\mathrm{MnO}$ in dark halos (Fig. 6) are due to the breakdown and replacement of olivine by relatively $\mathrm{Mg}$-poor, $\mathrm{K}$ - and Fe-rich celadonite and $\mathrm{Fe}$-oxyhydroxides. The $\mathrm{Fe}$-oxyhydroxides contain traces of $\mathrm{Mn}$ (Table 1), which likely account for the increased $\mathrm{MnO}$ in the 
Table 2. Whole-rock chemical analysis.

\begin{tabular}{|c|c|c|c|c|c|c|c|c|c|c|c|c|c|c|c|c|}
\hline Core-section & IR-1 & IR-2 & IR-3 & IR-3 & IR-4 & $1 R-4$ & IR-5 & IR-5 & $2 R-2$ & $2 \mathrm{R}-2$ & $3 R-1$ & $3 R-1$ & $4 \mathrm{R}-1$ & $4 \mathrm{R}-1$ & $4 \mathrm{R}-2$ & $4 \mathrm{R}-2$ \\
\hline Interval $(\mathrm{cm})$ & $65-67$ & $115-118$ & $47-50$ & $52-54$ & $99-101$ & $102-104$ & $36-38$ & $36-38$ & $15-17$ & $38-40$ & $17-19$ & $25-28$ & $12-15$ & $31-34$ & $21-23$ & $23-26$ \\
\hline Type & Dark gray & Brown & Gray & Dark halo & Brown & Gray & Gray & Dark halo & Gray & $\begin{array}{l}\text { Dark halo + } \\
\text { red band }\end{array}$ & Red halo & Gray & Dark halo & Gray & Dark halo & Gray \\
\hline Depth (mbsf) & 260.41 & 263.17 & 264.25 & 264.37 & 267.09 & 267.12 & 268.29 & 268.29 & 293.25 & 294.1 & 298.75 & 299.3 & 303.09 & 303.59 & 307.51 & 307.54 \\
\hline \multicolumn{17}{|l|}{$\mathrm{W} t \%$} \\
\hline $\mathrm{SiO}_{2}$ & 49.30 & 48.70 & 50.60 & 46.40 & 45.70 & 51.20 & 50.10 & 47.80 & 50.90 & 48.70 & 44.00 & 50.20 & 49.10 & 50.90 & 48.10 & 50.80 \\
\hline $\mathrm{Al}_{2} \mathrm{O}_{3}$ & 13.90 & 13.50 & 14.50 & 12.60 & 12.60 & 14.50 & 14.70 & 13.50 & 15.40 & 14.50 & 13.60 & 15.10 & 14.20 & 15.20 & 13.60 & 15.00 \\
\hline $\mathrm{M}_{8} \mathrm{O}$ & 7.26 & 5.82 & 6.37 & 5.68 & 5.58 & 6.55 & 6.66 & 5.90 & 6.04 & 5.21 & 4.70 & 5.87 & 5.87 & 6.20 & 5.79 & 6.91 \\
\hline $\mathrm{Fe}_{2} \mathrm{O}_{3} \mathrm{~T}$ & 12.60 & 14.00 & 9.44 & 15.40 & 16.60 & 9.52 & 9.76 & 15.00 & 9.96 & 13.20 & 18.70 & 11.10 & 13.30 & 8.99 & 14.50 & 9.10 \\
\hline $\mathrm{MnO}$ & 0.20 & 0.22 & 0.16 & 0.20 & 0.21 & 0.18 & 0.19 & 0.20 & 0.19 & 0.28 & 0.23 & 0.22 & 0.24 & 0.18 & 0.24 & 0.20 \\
\hline $\mathrm{CaO}$ & 11.10 & 10.80 & 11.50 & 11.60 & 10.90 & 11.30 & 11.80 & 10.60 & 10.00 & 9.78 & 9.51 & 9.40 & 10.80 & 12.00 & 11.30 & 11.90 \\
\hline $\mathrm{Na}_{2} \mathrm{O}$ & 2.56 & 2.60 & 2.70 & 2.42 & 2.56 & 2.78 & 2.89 & 2.50 & 3.11 & 3.38 & 2.84 & 3.46 & 2.53 & 2.62 & 2.41 & 2.66 \\
\hline $\mathrm{K}_{2} \mathrm{O}$ & 0.08 & 0.66 & 0.60 & 0.83 & 0.72 & 0.62 & 0.34 & 0.67 & 0.41 & 0.56 & 0.64 & 0.50 & 0.63 & 0.32 & 0.63 & 0.29 \\
\hline $\mathrm{TiO}_{2}$ & 1.82 & 1.83 & 1.90 & 1.59 & 1.67 & 1.88 & 1.91 & 1.78 & 2.05 & 1.97 & 1.86 & 2.10 & 1.97 & 2.07 & 1.87 & 2.05 \\
\hline $\mathrm{P}_{2} \mathrm{O}_{5}$ & 0.18 & 0.19 & 0.20 & 0.19 & 0.19 & 0.20 & 0.17 & 0.18 & 0.31 & 0.25 & 0.31 & 0.48 & 0.19 & 0.21 & 0.19 & 0.20 \\
\hline $\mathrm{H}_{2} \mathrm{O}^{+}$ & 0.90 & 1.20 & & 1.80 & 2.10 & & 1.40 & 1.60 & 1.80 & 2.00 & 2.40 & 1.60 & 1.50 & 0.60 & 1.50 & 1.40 \\
\hline $\mathrm{CO}_{2}$ & 0.03 & 0.15 & & 1.32 & 1.42 & & 0.04 & 0.05 & 0.13 & 0.40 & 1.39 & 0.19 & 0.04 & 0.15 & 0.30 & 0.06 \\
\hline LOI & 0.05 & 0.15 & 1.08 & 1.02 & 11.72 & 0.77 & 8.0 & 0.00 & 8.17 & 0.70 & ? & . & 8.07 & 年 & . & 0.00 \\
\hline Total & 99.29 & 99.09 & 99.05 & 99.56 & 99.81 & 99.50 & 99.45 & 99.27 & 99.90 & 99.73 & 99.84 & 99.82 & 99.83 & 98.95 & 99.85 & 100.06 \\
\hline $\mathrm{Fe}^{3+} / \mathrm{Fe}^{\mathrm{T}}$ & 0.49 & 0.59 & & 0.70 & 0.73 & & 0.48 & 0.66 & 0.56 & 0.62 & 0.82 & 0.64 & 0.59 & 0.46 & 0.60 & 0.44 \\
\hline $\mathrm{Fe}_{2} \mathrm{O}_{3}$ & 6.16 & 8.22 & & 10.73 & 12.16 & & 4.65 & 9.89 & 5.56 & 8.20 & 15.36 & 7.10 & 7.86 & 4.10 & 8.72 & 3.99 \\
\hline $\mathrm{FeO}$ & 5.80 & 5.20 & & 4.20 & 4.00 & & 4.60 & 4.60 & 4.00 & 4.50 & 3.00 & 3.60 & 4.90 & 4.40 & 5.20 & 4.60 \\
\hline \multicolumn{17}{|l|}{$\mathrm{ppm}$} \\
\hline $\mathrm{Ba}$ & 60 & 60 & 30 & 80 & 130 & 20 & 160 & 50 & 40 & 200 & 70 & 50 & 60 & 20 & 70 & 30 \\
\hline Co & 50 & 50 & & 50 & 40 & & 70 & 50 & 40 & 60 & 40 & 40 & 60 & 50 & 70 & 60 \\
\hline $\mathrm{Cr}$ & 120 & 110 & 110 & 110 & 110 & 110 & 170 & 110 & 90 & 160 & 110 & 80 & 110 & 130 & 120 & 120 \\
\hline $\mathrm{Cu}$ & 60 & 40 & & 20 & 30 & & 40 & 50 & 50 & 60 & 30 & 20 & 40 & 40 & 70 & 100 \\
\hline $\mathrm{Ni}$ & 50 & 40 & & 50 & 50 & & 70 & 40 & 50 & 50 & 60 & 30 & 40 & 60 & 40 & 70 \\
\hline $\mathrm{Rb}$ & 20 & 20 & 20 & 30 & 30 & 10 & 20 & 30 & 20 & 30 & 10 & 20 & 10 & 20 & 30 & 10 \\
\hline $\mathrm{Sr}$ & 110 & 110 & 130 & 110 & 110 & 120 & 130 & 120 & 140 & 150 & 120 & 140 & 120 & 140 & 110 & 160 \\
\hline $\mathrm{Y}$ & 30 & 30 & 30 & 30 & 20 & 30 & 30 & 30 & 60 & 60 & 40 & 80 & 30 & 50 & 30 & 40 \\
\hline $\mathrm{Zn}$ & 90 & 90 & & 100 & 100 & & 90 & 100 & 100 & 110 & 110 & 100 & 100 & 90 & 110 & 110 \\
\hline $\mathrm{Zr}$ & 130 & 130 & 120 & 110 & 120 & 110 & 120 & 140 & 200 & 160 & 170 & 200 & 130 & 130 & 110 & 130 \\
\hline
\end{tabular}

Note: Blanks = not analyzed. 
Table 3. Stable isotopic analyses of vein carbonates, Hole 843B.

\begin{tabular}{|c|c|c|c|c|c|}
\hline \multirow[b]{2}{*}{$\begin{array}{l}\text { Core, section, } \\
\text { interval }(\mathrm{cm})\end{array}$} & \multirow[b]{2}{*}{$\begin{array}{l}\text { Depth } \\
\text { (mbsf) }\end{array}$} & \multirow[b]{2}{*}{$\begin{array}{c}\delta^{13} \mathrm{C} \\
(\% \circ \text { PDB })\end{array}$} & \multirow[b]{2}{*}{$\begin{array}{c}\delta^{18} \mathrm{O} \\
(\% \text { SMOW })\end{array}$} & \multicolumn{2}{|c|}{$\begin{array}{l}\text { Equilibration } \\
\text { temperature }\end{array}$} \\
\hline & & & & $\begin{array}{l}0 \% \\
\text { fluid }\end{array}$ & $\begin{array}{l}-2 \% \\
\text { fluid }\end{array}$ \\
\hline \multicolumn{6}{|l|}{ 136-843B- } \\
\hline IR-3, 52-54 & 264.37 & 2.6 & 25.5 & 37 & 27 \\
\hline $1 \mathrm{R}-4,99-101$ & 267.09 & 2.8 & 30.5 & 13 & 5 \\
\hline $1 \mathrm{R}-6,7-9$ & 268.99 & 2.7 & 30.3 & 14 & 6 \\
\hline $1 \mathrm{R}-5,36-38$ & 268.90 & 2.4 & 29.4 & $\begin{array}{l}14 \\
18\end{array}$ & 10 \\
\hline $2 \mathrm{R}-1,115-118$ & 292.36 & 1.4 & 26.1 & 34 & 24 \\
\hline $2 \mathrm{R}-2,38-40$ & 294.10 & 2.7 & 28.7 & 21 & 13 \\
\hline $3 \mathrm{R}-1,17-19$ & 298.75 & 2.4 & 26.4 & 32 & 23 \\
\hline $4 \mathrm{R}-2,21-23$ & 307.51 & 2.4 & 27.1 & 29 & 20 \\
\hline
\end{tabular}

${ }^{a}$ Equilibration temperatures calculated assuming formation in equilibrium with water having $\delta^{18} \mathrm{O}$ of normal seawater $\left(0 \%\right.$ ) and ${ }^{18} \mathrm{O}$-depleted pore fluids $(-2 \%)$ using fractionation factors of O'Neil et al. (1969).

dark halos. The decreases in $\mathrm{TiO}_{2}$ contents of the dark halos (Fig. 6) are difficult to explain, because celadonite and $\mathrm{Fe}$-oxyhydroxides in these zones tend to contain greater amounts of $\mathrm{Ti}$ than the saponites in the gray host rocks (Fig. 7), which have higher $\mathrm{TiO}_{2}$ contents. Perhaps the high Ti contents of phyllosilicates and oxides in the dark halos simply indicate that Ti was mobile in these zones: Ti may have been lost from these zones and incorporated into phyllosilicates and oxides filling adjacent fractures. The losses of $\mathrm{Al}_{2} \mathrm{O}_{3}$ and $\mathrm{Na}_{2} \mathrm{O}$ from dark halos may be due to the slight alteration of plagioclase and glassy groundmass material, whereas $\mathrm{CaO}$ was retained in some samples as secondary calcite. Celadonites have a range of $\mathrm{Al}$ contents, but saponites have consistently high Al contents (Fig. 4), indicating that saponite in the gray host rocks was more efficient at retaining $\mathrm{Al}$ than celadonite in the dark halos.

Dark alteration halos containing celadonite or celadonite-smectite occur in otherwise unaltered very young $(<1 \mathrm{Ma})$ seafloor rocks (Humphris et al., 1980; Laverne and Vivier, 1983: Adamson and Richards, 1990). The similarity of the mineralogy and occurrence of these alteration zones to the celadonite-bearing dark halos around veins in Hole 843B suggests that the latter similarly formed very early during the history of the site.

The switch from formation of celadonite to that of Fe-oxyhydroxides likely reflects an increase in oxygen fugacity and relatively open circulation of cold, oxidizing seawater. Formation of the narrow red halos and the wider brown oxidation zones in highly fractured and veined Sections 136-843B-1R-3 and -4 occurred at this time, resulting in increased oxidation and higher $\mathrm{H}_{2} \mathrm{O}^{+}$contents of these rocks (Fig. 6).

The gray host rocks have lower $\mathrm{K}_{2} \mathrm{O}$ contents and are less oxidized than adjacent dark halos, indicating lower seawater/rock ratios in the gray host rocks. Saponite contains mostly ferrous iron (Andrews et al., 1983 ) and is associated with pyrite in veins of Hole 843B, implying more reducing conditions during saponite formation than during earlier celadonite and Fe-oxyhydroxide formation. This change is probably related to more restricted circulation of seawater, possibly due to burial of the crust by sediments. Saponite can form in seafloor basalts at temperatures of up to $100^{\circ}-170^{\circ} \mathrm{C}$ (Stakes and O'Neil, 1984; Alt, Muehlenbachs, and Honnorez, 1986), but various evidence (shallow depth of the cores; abundant $\mathrm{Fe}$-oxyhydroxides indicating circulation of cold seawater; temperatures estimated from $\delta^{18} \mathrm{O}$ of calcites in Table 3 ) suggest that saponite probably formed at much lower temperatures of $0^{\circ}-50^{\circ} \mathrm{C}$ in Hole $843 \mathrm{~B}$ (e.g., Böhlke et al., 1984). Saponite is absent in young (less than $1 \mathrm{Ma}$ ) rocks from the Pacific, but is present in rocks greater than about 2.5 million years old (Humphris et al., 1981; Laverne and Vivier, 1983). This sequence is consistent with that observed in rocks from Hole $843 \mathrm{~B}$, and gives some idea of the possible timing of saponite formation in Hole 843B.

Despite formation of a Mg-rich secondary phase (saponite) in the gray host rocks, there is no evidence for $\mathrm{Mg}$-enrichment of the rocks,

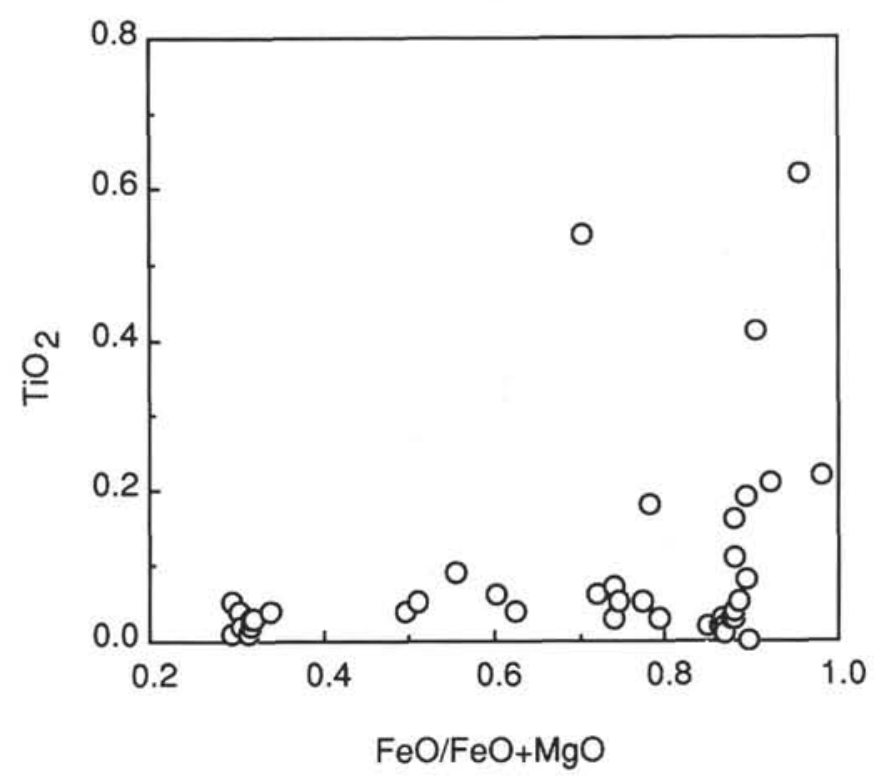

Figure 7. $\mathrm{TiO}_{2}$ contents vs. $\mathrm{FeO} / \mathrm{FeO}+\mathrm{MgO}$ of secondary phyllosilicates (wt\%). Celadonite and $\mathrm{Fe}$-oxyhydroxide mixtures with high $\mathrm{FeO} / \mathrm{FeO}+\mathrm{MgO}$ have high $\mathrm{TiO}_{2}$ contents, whereas saponites at lower $\mathrm{FeO} / \mathrm{FeO}+\mathrm{MgO}$ have low $\mathrm{TiO}_{2}$ contents.

and $\mathrm{Mg}$ was actually lost from the adjacent dark halos and brown zones. $\mathrm{Mg}$ may simply have been redistributed on a local scale, with $\mathrm{Mg}$ lost from individual rocks precipitated as saponite filling fractures in the rocks. Such fixation of $\mathrm{Mg}$ in veins can include seawaterderived $\mathrm{Mg}$ and actually lead to a net increase in the $\mathrm{Mg}$ content of the 500- to 1000-m-thick oceanic volcanic section as a whole (Andrews, 1980; Stakes and Scheidegger, 1981; Alt, Honnorez, Laverne, and Emmermann, 1986).

The formation of blue-green celadonite in Hole 843B followed that of earlier green celadonite and $\mathrm{Fe}$-oxyhydroxides. This sequence suggests either that fluctuations in solution composition caused alternation between formation of celadonite and Fe-hydroxides, or that a distinct second stage of celadonite formation occurred. Various evidence suggests that the latter case is more likely: the blue-green celadonite is optically and chemically distinguishable from the earlier green celadonite, indicating a change or evolution in solution composition rather than simple fluctuations; the blue-green celadonite veins also cut across and fill reopened and brecciated green celadonite + Fe-oxyhydroxide veins, consistent with sealing of fractures and subsequent re-fracturing of the rocks at a later stage; and the late bluegreen celadonite occurs both before and after calcite, which is itself the last phase to form in Hole 843B and elsewhere in altered oceanic volcanics (Staudigel et al., 1981; Alt and Honnorez, 1984; Böhlke et al., 1984; Alt, Honnorez, Laverne, and Emmermann, 1986; Alt et al., 1992). The absolute timing of these different celadonite stages is unknown, however, but could be important in interpreting $\mathrm{K} / \mathrm{Ar}$ dates for celadonites in oceanic crust (e.g., Staudigel et al., 1986). Celadonitic phyllosilicates in altered seafloor basalts are generally interpreted as early-forming phases (Laverne and Vivier, 1983; Alt and Honnorez, 1984; Böhlke et al., 1984; Adamson and Richards, 1989), but celadonite has also been described as a late-forming phase (Stakes and Scheidegger, 1981).

The last phase to form in basalts from Hole 843B was calcite ( \pm pyrite) in crosscutting veins and filling pores in the rocks. Such late-stage calcite formation is typical in altered seafloor volcanics, and is interpreted to occur at low seawater/rock ratios from highly evolved, Mg-depleted fluids (Alt and Honnorez, 1984; Böhlke et al., 1984; Alt, Honnorez, Laverne, and Emmermann, 1986; Alt et al., 


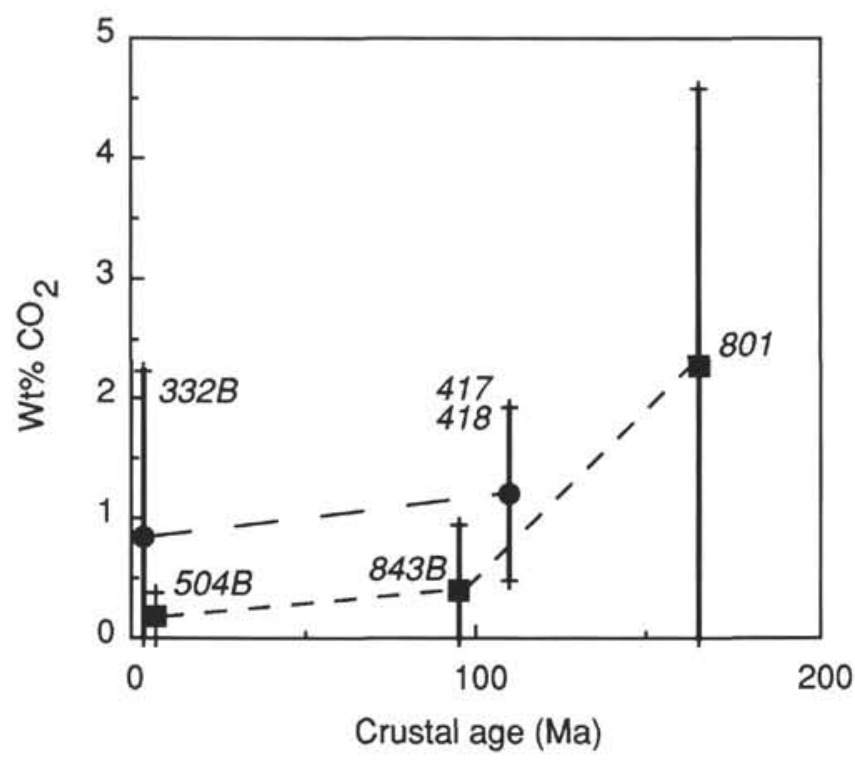

Figure 8. $\mathrm{CO}_{2}$ contents of some DSDP/ODP volcanic sections vs. age of crust. Square $=$ Pacific crust; circles $=$ Atlantic crust ; plotted symbols show mean and standard deviation for whole-rock analyses. Data for Sites 417 and 418 from Alt and Honnorez (1984); Site 504 from Alt, Honnorez, Laverne, and Emmermann (1986); Site 801 from Alt et al. (1992); Site 332B from Aumento, Melson, et al. (1977).

1992). On the basis of comparison of long volcanic sections at two DSDP drill sites in 3.2- and 110-Ma Atlantic crust (Sites 332 and 418; Fig. 8), Staudigel et al. (1981) suggested that the $\mathrm{CO}_{2}$ (calcite) content of ocean crust increases with time as the crust ages. Comparison of calcite ${ }^{87} \mathrm{Sr} /{ }^{86} \mathrm{Sr}$ ratios to the values for seawater through time constrained calcite formation to within a few tens of million years of crustal formation, however (Staudigel et al., 1981). Data for some Pacific crust are shown along with the Atlantic sites in Figure 8. There is apparently a progressive increase in $\mathrm{CO}_{2}$ content from younger to older crust in the Pacific, suggesting the possibility of prolonged or very late-stage calcite formation (i.e., more than $100 \mathrm{Ma}$ after crustal generation). Multiple stages of late crosscutting calcite veins are present in some samples from Hole 843B, and are common in 170 Ma crust from Site 801 (Alt et al., 1992), consistent with the hypothesized continued precipitation of calcite in the crust.

Some $\mathrm{CO}_{2}$ variations, like that between Sites 332 and 504, may represent heterogeneities in the amount of late fracturing and calcite formation in the volcanics, which are likely related to local volcanic and tectonic activity and may not be significant as temporal variations. The $\mathrm{CO}_{2}$ contents at Site 801 decrease significantly below about 100 mbsf, but the drilled section only extends to $150 \mathrm{~m}$, so the average for this site plotted in Figure 8 may be biased toward high values in the upper $100 \mathrm{~m}$ of the crust. Also, the scatter in the $\mathrm{CO}_{2}$ content data at each site is large, contributing to the high standard deviations in Figure 8. Much of the $\mathrm{CO}_{2}$ in altered crust may reside in veins rather than as pore space fillings and pseudomorphs of primary silicate minerals in the altered rocks (Staudigel et al., 1990), so the data used in Figure 8 may not be entirely representative. Nevertheless, the observations of multiple late-stage crosscutting calcite veins plus the increase in wholerock $\mathrm{CO}_{2}$ contents shown in Figure 8 suggest a trend that is worthy of further investigation. Quantification of the amount of carbonate fixed in veins for the various sites, plus dating of crosscutting calcite veins would allow a better evaluation of progressive fixation of $\mathrm{CO}_{2}$ in oceanic crust, but are beyond the scope of this paper.

On the basis of high $\mathrm{K}_{2} \mathrm{O}$ contents on plots of $\mathrm{K}_{2} \mathrm{O}$ vs. $\mathrm{TiO}_{2}$ and $\mathrm{MgO}$, it was suggested that some basalts from Hole $843 \mathrm{~B}$ could either be enriched MORB (E-MORB) or altered N-MORB (Dziewonski, Wilkens, Firth, et al., 1992). On plots of $\mathrm{K}_{2} \mathrm{O}$ vs. $\mathrm{TiO}_{2}$ and $\mathrm{MgO}$ the gray host rocks of this study plot close to or within the N-MORB field, whereas the dark halos define alteration trends to higher $\mathrm{K}_{2} \mathrm{O}$ and lower $\mathrm{TiO}_{2}$ and $\mathrm{MgO}$ contents (Fig. 9). E-MORB and transitional MORB (T-MORB) are characterized by higher $\mathrm{K}_{2} \mathrm{O}$ and $\mathrm{TiO}_{2}$ than $\mathrm{N}-\mathrm{MORB}$, but also by higher $\mathrm{K} / \mathrm{Ti}$ ratios (Sinton et al., 1991). K/Ti ratios of Hole $843 \mathrm{~B}$ rocks show a trend to higher values in the dark gray and brown alteration halos (Fig. 9). The above data suggest that some of the T-MORB characteristics (high $\mathrm{K}_{2} \mathrm{O}$, high $\mathrm{K} / \mathrm{Ti}$ ) of the rocks from Hole $843 \mathrm{~B}$ could conceivably be caused by alteration of $\mathrm{N}-\mathrm{MORB}$. Although $\mathrm{Ba}$ and $\mathrm{Zr}$ may be slightly affected by alteration, the uniformly high $\mathrm{Ba}$ and $\mathrm{Zr}$ contents of Hole $843 \mathrm{~B}$ lavas are more consistent with T-MORB than N-MORB compositions (Table 2), and the $\mathrm{K} / \mathrm{Ti}$ ratios of least altered gray zones fall in the range for T-MORB (Sinton et al., 1991). Thus, the data indicate that although seawater interaction has significantly affected the composition of the more altered samples (dark gray and brown zones), the T-MORB primary compositions can still be observed in the less-altered gray host rocks. On the basis of high $\mathrm{K} / \mathrm{Ti}$ and $\mathrm{K} / \mathrm{P}$ ratios, moderate $(\mathrm{La} / \mathrm{Sm})_{\mathrm{N}}$ ratios, and low $\mathrm{Y} / \mathrm{Nb}$ and $\mathrm{Zr} / \mathrm{Nb}$ ratios King et al. (this volume) conclude that Hole 843 B rocks are mainly T-MORB.

\section{CONCLUSIONS}

Basalts from the 70.9-m basement section of Hole 843B, located in 95-Ma crust of the Hawaiian Arch, exhibit the effects of low-temperature alteration by seawater. Millimeter- to centimeter-sized dark alteration halos around veins are $5 \%-15 \%$ altered, mainly with olivine replaced and pores filled with celadonite and $\mathrm{Fe}$-oxyhydroxides plus minor saponite and calcite. Adjacent gray host rocks are about $15 \%$ altered, with olivine replaced and pores filled by saponite and calcite. The dark halos are enriched in $\mathrm{H}_{2} \mathrm{O}^{+}, \mathrm{CO}_{2}, \mathrm{Fe}_{2} \mathrm{O}_{3}{ }^{\mathrm{T}}, \mathrm{K}_{2} \mathrm{O}, \mathrm{MnO}$, and $\mathrm{Fe}^{3+} / \mathrm{Fe}^{\mathrm{T}}$ and depleted in $\mathrm{SiO}_{2}, \mathrm{Al}_{2} \mathrm{O}_{3}, \mathrm{MgO}$ and $\mathrm{TiO}_{2}$ relative to gray host rocks. The gray host rocks more closely reflect unaltered rock compositions, but are nevertheless enriched in $\mathrm{H}_{2} \mathrm{O}^{+}, \mathrm{CO}_{2}$, and $\mathrm{K}_{2} \mathrm{O}$, and have elevated $\mathrm{Fe}^{3+} / \mathrm{Fe}^{\mathrm{T}}$. Brown alteration halos occur around veins where veins are more abundant and are similar to dark halos, but contain more $\mathrm{Fe}$-oxyhydroxides and exhibit greater $\mathrm{Fe}_{2} \mathrm{O}_{3}{ }^{\mathrm{T}}$ contents and higher $\mathrm{Fe}^{3+} / \mathrm{Fe}^{\mathrm{T}}$. Trends to higher $\mathrm{K}_{2} \mathrm{O}$ and $\mathrm{K} / \mathrm{Ti}$ in Hole $843 \mathrm{~B}$ samples are attributed to alteration, but the generally high $\mathrm{Ba}$ and $\mathrm{Zr}$ contents and the high $\mathrm{K} / \mathrm{Ti}$ in least altered gray rocks are consistent with T-MORB primary compositions. Stable isotopic compositions of vein carbonates are consistent with their precipitation from seawater at temperatures of $5^{\circ}-40^{\circ} \mathrm{C}$.

Crosscutting relationships of veins and zoned vein and vesicle fillings reveal a sequence of secondary mineral formation and alteration conditions. Celadonite and Fe-oxyhydroxides formed under oxidizing conditions at low temperatures $\left(<50^{\circ} \mathrm{C}\right)$. The dark alteration halos where these phases occur formed relatively early, probably within $1 \mathrm{Ma}$ of crustal formation. Saponite formed later, at lower seawater/rock ratios and under more reducing conditions as seawater circulation became more restricted. Calcite and pyrite formed last in veins and vesicles from more evolved, seawater-derived fluids at temperatures of $5^{\circ}-40^{\circ} \mathrm{C}$. These effects are similar to those in other ODP/DSDP cores, but a second stage of celadonite, with compositions distinct from the early celadonite, also occurred relatively late (within the "calcite stage") in Hole $843 \mathrm{~B}$, and may be related to refracturing of the crust and introduction of less-evolved seawater solutions into the rocks.

Abundant calcite, particularly in brecciated zones, characterizes Hole $843 \mathrm{~B}$ rocks as well as other old ocean crust. The mean bulk $\mathrm{CO}_{2}$ contents of DSDP/ODP cores exhibit a general increase with age suggesting progressive fixation of $\mathrm{CO}_{2}$ as calcite in the crust, but this could be complicated by local heterogeneities in fracturing and consequent calcite formation in the crust. 

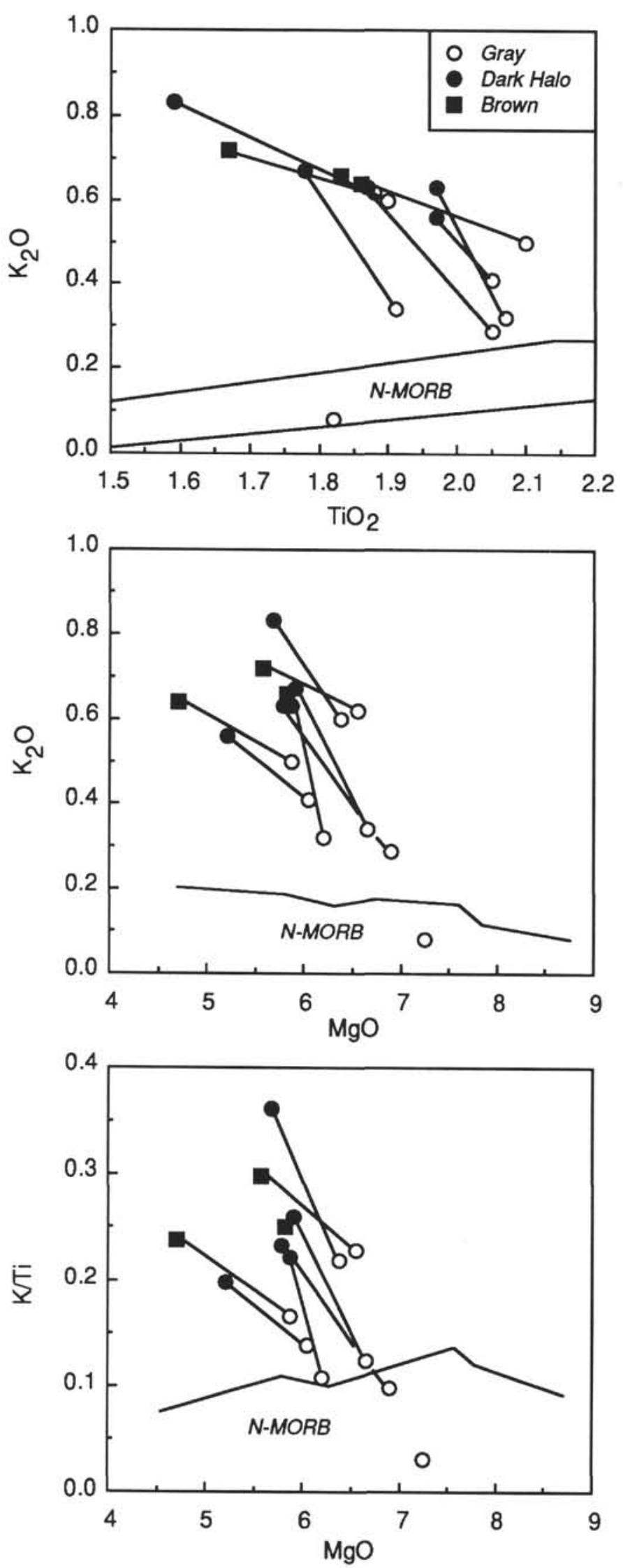

Figure 9. Alteration trends of basalts from Hole 843B compared to N-MORB compositions. N-MORB fields from Natland and Melson (1980) and Sinton et al. (1991). Data in wt\%.

\section{ACKNOWLEDGMENTS}

This work was supported by a grant from JOI-USSAC. The author thanks Roy Wilkens for initiating this study and K.C. Lohman for providing stable isotopic analyses of carbonates. Reviews by Kathryn Gillis and Peter Schiffman and comments by John Bender helped to improve the paper.

\section{REFERENCES"}

Adamson, A.C., and Richards, H.G., 1990. Low-temperature alteration of very young basalts from ODPHole 648B: Serocki volcano, Mid-Atlantic Ridge. In Detrick, R., Honnorez, J., Bryan, W.B., Juteau, T., et al. Proc. ODP, Sci. Results, 106/109: College Station, TX (Ocean Drilling Program), 181-194.

Alt, J.C., and Honnorez, J., 1984. Alteration of the upper oceanic crust, DSDP Site 417: mineralogy and chemistry. Contrib. Mineral. Petrol., 87:149-169.

Alt, J.C., Honnorez, J., Laverne, C., and Emmermann, R., 1986. Hydrothermal alteration of a $1 \mathrm{~km}$ section through the upper oceanic crust, Deep Sea Drilling Project Hole 504B: mineralogy, chemistry, and evolution of seawater-basalt interactions. J. Geophys. Res., 91:10309-10335.

Alt, J.C., Lanord, C.F., Floyd, P., Castillo, P., and Galey, A., 1992. Low-temperature hydrothermal alteration of Jurassic ocean crust, ODP Site 801. In Larson, R.L., Lancelot, Y., et al., Proc. ODP, Sci. Results, 129: College Station, TX (Ocean Drilling Program), 415-427.

Alt, J.C., Muehlenbachs, K., and Honnorez, J., 1986. An oxygen isotopic profile through the upper kilometer of the oceanic crust, DSDP Hole 504B. Earth Planet. Sci. Lett., 80:217-229.

Andrews, A.J., 1977. Low-temperature fluid alteration of oceanic layer 2 basalts, DSDP Leg 37. Can. J. Earth Sci., 14:911-926.

, 1980. Saponite and celadonite in Layer 2 basalts, DSDP Leg 37. Contrib. Mineral. Petrol., 73:323-340.

Andrews, A.J., Dollase, W.A., and Fleet, M.E., 1983. A Mossbauer study of saponite in Layer 2 basalts, Deep Sea Drilling Program Leg 69. In Cann, J.R., Langseth, M.G., Honnorez, J., Von Herzen, R.P., White, S.M., et al., Init. Repts. DSDP, 69: Washington (U.S. Govt. Printing Office), 585-588.

Aumento, F., Melson, W.G., et al., 1977. Init. Repts. DSDP, 37: Washington (U.S. Govt. Printing Office).

Böhlke, J.K., Alt, J.C., and Muehlenbachs, K., 1984. Oxygen isotope-water relations in altered deep-sea basalts: low temperature mineralogical controls. Can. J. Earth Sci., 21:67-77.

Dziewonski, A., Wilkens, R., Firth, J., et al., 1992. Proc. ODP, Init. Repts., 136: College Station, TX (Ocean Drilling Program).

Humphris, S.E., Melson, W.G., and Thompson, R.N., 1980. Basalt weathering on the East Pacific Rise and Galapagos Spreading Center, DSDP Leg 54. In Rosendahl, B.R., Hekinian, R., et al., Init. Repts. DSDP, 54: Washington (U.S. Govt. Printing Office), 773-787.

Kastner, M., and Gieskes, J.M., 1976. Interstitial water profiles and sites of diagenetic reactions, Leg 35. DSDP, Bellingshausen Abyssal Plain. Earth Planet. Sci. Lett., 33:11-20.

Laverne, C., and Vivier, G., 1983. Petrographical and chemical study of basement from the Galapagos Spreading Center, Leg 70. In Honnorez, J., Von Herzen, R.P., et al., Init. Repts. DSDP, 70: Washington (U.S. Govt. Printing Office), 375-390.

Lawrence, J.R., and Gieskes, J.M., 1981. Constraints on water transport and alteration in the oceanic crust from the isotopic composition of pore water. J. Geophys. Res., 86:7924-7934.

Natland, J.H., and Melson, W.G., 1980. Compositions of basaltic glasses from the East Pacific Rise and Siqueiros fracture zone, near $9^{\circ} \mathrm{N}$. In Rosendahl, B.R., Hekinian, R., et al., Init. Repts. DSDP. 54: Washington (U.S. Govt. Printing Office), 705-723.

O’Neil, J.R., Clayton, R.N., and Mayeda, T.K., 1969. Oxygen isotope fractionation in divalent metal carbonates. J. Chem. Phys., 51:5547-5558.

Petersen, N., Eisenach, P., and Bleil, U., 1979. Low temperature alteration of the magnetic minerals in ocean floor basalts. In Talwani, M., Harrison, C.G., and Hayes, D. (Eds.), Deep Drilling Results in the Atlantic Ocean: Ocean Crust. Am. Geophys. Union, Maurice Ewing Ser., 2:169-209.

Seyfried, W.E., Jr., Shanks, W.C., III, and Dibble, W.E., Jr., 1978. Clay mineral formation in DSDP Leg 34 basalt. Earth Planet. Sci. Lett., 41:265-276.

Shackleton, N.J., and Kennett, J.P., 1975. Paleotemperature history of the Cenozoic and the initiation of Antarctic glaciation: oxygen and carbon

- Abbreviations for names of organizations and publications in ODP reference lists follow the style given in Chemical Abstracts Service Source Index (published by American Chemical Society). 
isotope analyses in DSDP Sites 277.279, and 281. In Kennett, J.P., Houtz, R.E., et al., Init. Repts. DSDP, 29: Washington (U.S. Govt. Printing Office), $743-755$.

Sinton, J.M., Smaglik, S.M., Mahoney, J.J., and Macdonald, K.C., 1991. Magmatic processes at superfast spreading mid-ocean ridges. J. Geophys. Res., 96:6133-6155.

Stakes, D.S., and O'Neill, J.R., 1982. Mineralogy and stable isotope geochemistry of hydrothermally altered oceanic rocks. Earth Planet. Sci. Lett., 57:285-304.

Stakes, D.S., and Scheidegger, K.F., 1981. Temporal variations in secondary minerals from Nazca Plate basalts, In Kulm, L.D. (Ed.), Nazca Plate: Crustal Formation and Andean Convergence. Mem.-Geol. Soc. Am., 154:109-130.

Staudigel, H., Gillis, K., and Duncan, R., 1986. K/Ar and Rb/Sr ages of celadonites from the Troodos ophiolite, Cyprus. Geology, 14:72-75.
Staudigel, H., Hart, S.R., and Richardson. S.H., 1981. Alteration of the oceanic crust: processes and timing. Earth Planet. Sci. Lett., 52:311-327.

Staudigel, H.R., Hart, S.R., Schmincke, H.U., and Smith, B.M., 1990. Cretaceous ocean crust at DSDP Sites 417 and 418: carbon uptake from weathering versus loss by magmatic outgassing. Geochim. Cosmochim. Acta, 53:3091-3094.

Winterer, E.L., Ewing, J.I., et al., 1973. Init. Repts. DSDP, 17: Washington (U.S. Govt. Printing Office).

Date of initial receipt: 28 August 1992

Date of acceptance: 15 December 1992

Ms 136SR-214 